\title{
Probabilistic Optical and Radar Initial Orbit
}

\section{Determination}

\author{
Roberto Armellin ${ }^{1}$ \\ University of Surrey, Guildford, GU2 7XH, United Kingdom \\ Pierluigi Di Lizia ${ }^{2}$ \\ Politecnico di Milano, Milano, 20156, Italy
}

Future space surveillance requires dealing with uncertainties directly in the initial orbit determination phase. We propose an approach based on Taylor differential algebra to both solve the initial orbit determination (IOD) problem and to map uncertainties from the observables space into the orbital elements space. This is achieved by approximating in Taylor series the general formula for probability density function (pdf) mapping through nonlinear transformations. In this way the mapping is obtained in an elegant and general fashion. The proposed approach is applied to both angles-only and two position vectors IOD for objects in LEO and GEO.

\section{Introduction}

The total number of active satellites, rocket bodies, and debris larger than $10 \mathrm{~cm}$ is currently about 20,000. Considering all resident space objects larger than $1 \mathrm{~cm}$ this rises to an estimated minimum of 500,000 objects. Latest generation sensor networks will produce millions of observations per day $[1,2]$. Identifying observations belonging to the same object will be one of the main challenges of orbit determination. One requirement to perform reliable data association is to have realistic uncertainties for initial orbit solutions, which could also be used to initialize Bayesian estimators for orbit refinement [3]. Meeting this requirement demands two main steps: solving the

\footnotetext{
1 Senior Lecturer, Surrey Space Centre, BA Building; r.armellin@surrey.ac.uk.

2 Assistant Professor, Department of Aerospace Science and Technology, via La Masa 34; pierluigi.dilizia@polimi.it.
} 
initial orbit determination (IOD) problem, and mapping the error distributions from observations to the initial orbit solutions. The second step is the main focus of this work.

In IOD, the number of observations is equal to the number of unknowns, and thus a nonlinear system of equations is to be solved. Moreover, simplified dynamical models are often used (e.g. Keplerian motion) and measurement errors are not taken into account (the problem is deterministic). Based on the set of available measurements, different IOD problems can be framed. In this work we consider two cases: angles-only and two position vectors IOD. In angles-only IOD a resident space object (RSO) is observed three times with optical sensors on a single passage. Angles-only IOD is an old problem. Gauss' [4] and Laplace's [5] methods are commonly used to determine a Keplerian orbit that fits with three astrometric observations. These methods have been revisited and analyzed by a large number of authors [6-8], and new ones introduced more recently. The Double r-iteration technique of Escobal [9] and the approach of Gooding [10] are two examples of anglesonly IOD methods for RSO, whereas a procedure to correct the deterministic solution provided by the classical IOD algorithms has been introduced by Hussein et al. [11] to improve the performance of observation-to-observation association schemes. The admissible region methods, which were originally developed by Milani et al. [12], provide an alternative approach to the representation of the solution of the IOD problem $[13,14]$. In the two position vectors problem, two range measurements are obtained with a radar together with the information on instrument pointing angles. In this case, the classical Lambert problem can be formulated [15]. Although this problem was solved more than 200 years ago, many researchers are still working on devising robust and efficient resolution procedures $[16,17]$.

In general, the solution of both angles-only and two position vectors IOD is numerical and thus an explicit function that relates the observations with the object orbital parameters is not available. The classical methods proposed in the literature to map observation statistics into initial orbit statistics rely on linearizations [18]. However, the validity of the linearity assumption is often called into question due to the inherent nonlinearity of the mapping from the measurements space to the orbital space. Consequently, alternative methods have been recently devised to deal with nonlinearities, such as the use of particle based approaches [19-22] or the recent technique of the 
transformation of variables to map probability density functions from a specified domain into a different domain $[23,24]$. The method proposed in this work shares the same objective of the latter, with the difference that differential algebra (DA) is used to compute a semi-analytical representation of the mapping between the initial and the desired domains.

In this work, the relation between the observations and the object orbital parameters is approximated in terms of its high order Taylor expansion by using DA [25]. DA is used to solve the nonlinear equations associated to the IOD problem as well as to expand their solution in Taylor series with respect to the measurements. The resulting high-order map is referred to as the observations-to-solution (O2S) map. The O2S map can be profitably used to map analytically samples from the observation space into the RSO orbital parameters space, thus enabling for example efficient Monte Carlo (MC) simulations. In addition, for given statistical properties of the measurements, the availability of a Taylor approximation of the $\mathrm{O} 2 \mathrm{~S}$ allows one to obtain the statistical moments of the states by computing the expectation of its monomials, thus avoiding the need of samples $[26,27]$. More importantly, the O2S map (and the Taylor representation of its inverse, the $\mathrm{S} 2 \mathrm{O}$ map) can be manipulated to analytically represent the probability density function (pdf) of the IOD solution for any arbitrary pdf of the measurements. This is achieved by expanding in Taylor series the determinant of the Jacobian of the O2S map and using well known formula for pdf transformation $[28,29]$. This approach is independent on the representation of the dynamics and thus the Keplerian approximation can be removed.

The paper is organised as follows. An introduction on DA is given first, together with the algorithms for the expansion of the solution of implicit equations and the solution of a system of ODE. This section ends with the description of the procedure to select the expansion order. Section III describes the use of DA for mapping a pdf in a general fashion, using the Cartesian to polar transformation as an illustrative case. The method is then detailed in Sec. IV for the specific application of angles-only and two position vectors IOD. The optical observation of GEO spacecraft and the radar observation of an RSO in LEO are used as test cases in Sec. V to investigate the method's features and performance. Some final remarks conclude the paper. 


\section{Differential Algebra tools}

DA supplies the tools to compute the derivatives of functions within a computer environment [25]. More specifically, by substituting the classical implementation of real algebra with the implementation of a new algebra of Taylor polynomials, any deterministic function $f$ of $v$ variables that is $\mathcal{C}^{k+1}$ in the domain of interest $[-1,1]^{v}$ (these properties are assumed to hold for any function dealt with in this work) is expanded into its Taylor polynomial up to an arbitrary order $k$ with limited computational effort. In addition to basic algebraic operations, operations for differentiation and integration can be easily introduced in the algebra, thusly finalizing the definition of the differential algebraic structure of DA [30, 31]. Similarly to algorithms for floating point arithmetic, various algorithms were introduced in DA, including methods to perform composition of functions, to invert them, to solve nonlinear systems explicitly, and to treat common elementary functions [32]. The DA used for the computations in this work was implemented in the softwares COSY INFINITY [33] and DACE [34]. The reader may refer to [35] for the DA notation adopted throughout the paper.

\section{A. High-order expansion of the solution of ODE}

An important application of DA is the automatic computation of the high order Taylor expansion of the solution of an ODE with respect to either the initial conditions or any parameter of the dynamics $[32,35]$. This can be achieved by replacing the classical floating point operations of the numerical integration scheme, including the evaluation of the right hand side, by the corresponding DA-based operations. This way, starting from the DA representation of the initial condition $x_{0}$, the DA-based ODE integration supplies the Taylor expansion of the flow in $x_{0}$ at all the integration steps, up to any final time $t_{f}$. Any explicit ODE integration scheme can be adapted to work in the DA framework in a straightforward way. For the numerical integrations presented in this paper, a DA version of a 7/8 Dormand-Prince (8-th order solution for propagation, 7-th order solution for step size control) Runge-Kutta scheme is used. The main advantage of the DA-based approach is that there is no need to write and integrate variational equations to obtain high order expansions of the flow. It is therefore independent on the particular right hand side of the ODE and the method is quite efficient in terms of computational cost. 


\section{B. Expansion of the solution of parametric implicit equations}

Well-established numerical techniques (e.g., Newton's method) exist to compute numerically the solution of an implicit equation

$$
\boldsymbol{h}(\boldsymbol{y})=0
$$

with $\boldsymbol{h}: \mathbb{R}^{n} \rightarrow \mathbb{R}^{n}$. Suppose the vector function $\boldsymbol{h}$ depends explicitly on a vector of parameters $\boldsymbol{x}$, which yields the parametric implicit equation

$$
\boldsymbol{h}(\boldsymbol{y}, \boldsymbol{x})=0 .
$$

The solution of $(2)$ is the function $\boldsymbol{y}=\boldsymbol{f}(\boldsymbol{x})$ that solves the equality for any value of $\boldsymbol{x}$.

DA techniques can effectively handle the previous problem by representing $\boldsymbol{f}(\boldsymbol{x})$ in terms of its Taylor expansion with respect to $\boldsymbol{x}$. This result is achieved by applying partial inversion techniques as detailed in Di Lizia et al. [35]. The final result is

$$
\boldsymbol{y}=\mathcal{T}_{\boldsymbol{f}}^{k}(\boldsymbol{x})
$$

which is the $k$-th order Taylor expansion of the solution of the implicit equation. For every value of $\boldsymbol{x}$, the approximate solution of $\boldsymbol{h}(\boldsymbol{y}, \boldsymbol{x})=0$ can be easily computed by evaluating the Taylor polynomial (3). Apparently, the solution obtained by means of the polynomial map (3) is a Taylor approximation of the exact solution of Eq. (2). The accuracy of the approximation depends on both the order of the Taylor expansion and the displacement of $\boldsymbol{x}$ from its reference value.

The capability of expanding the solution of parametric implicit equations (PIE) is of key importance for this work, as these appear in both Lambert's and Kepler's problems, used here to solve the IOD problems and to map the resulting solution manifold forward in time when the two-body approximation is adopted. More importantly, any IOD problem can be framed as a PIE problem. The role of the vector of parameters $\boldsymbol{x}$ in Eq. (2) is played by the measurements, whereas $\boldsymbol{y}$ is the spacecraft state and $\boldsymbol{h}(\boldsymbol{y}, \boldsymbol{x})$ is the function of the residuals. Within this framework, the resulting polynomial $\mathcal{T}_{\boldsymbol{f}}^{k}$ in Eq. (3) maps analytically any variation of the measurements into the corresponding variation of the orbital state. Apparently, $\mathcal{T}_{\boldsymbol{f}}^{k}$ is the O2S map defined in Sec. I. 


\section{Kepler's problem}

In Kepler's problem the initial position and velocity vectors, $\boldsymbol{r}_{1}$, and $\boldsymbol{v}_{1}$ of an object are known at an epoch $t_{1}$. The problem consists in finding the position and velocity vectors of the body, $\boldsymbol{r}_{2}$ and $\boldsymbol{v}_{2}$, at epoch $t_{2}$ in the two-body approximation. This is done by computing the Lagrangian coefficient $f, f^{t}$ and $g, g^{t}$ at time $t_{2}$ and the new object state as

$$
\begin{aligned}
& \boldsymbol{r}_{2}=f_{2} \boldsymbol{r}_{1}+g_{2} \boldsymbol{v}_{1} \\
& \boldsymbol{v}_{2}=f_{2}^{t} \boldsymbol{r}_{1}+g_{2}^{t} \boldsymbol{v}_{1}
\end{aligned}
$$

For ellipses, the Langrangian coefficients are function of the eccentric anomaly difference $E_{2}-E_{1}$ and assume the form [15]

$$
\begin{aligned}
f_{2} & =1-\frac{a}{r_{1}}\left[1-\cos \left(E_{2}-E_{1}\right)\right] \\
g_{2} & =\frac{a \sigma_{1}}{\sqrt{\mu}}\left[1-\cos \left(E_{2}-E_{1}\right)\right]+r_{1} \sqrt{\frac{a}{\mu} \sin \left(E_{2}-E_{1}\right)} \\
f_{2}^{t} & =-\frac{\mu a}{r_{2} r_{1}} \sin \left(E_{2}-E_{1}\right) \\
g_{2}^{t} & =1-\frac{a}{r_{2}}\left[1-\cos \left(E_{2}-E_{1}\right)\right]
\end{aligned}
$$

where $a$ is the semi-major axis of the orbit, $\mu$ the gravitational parameter, $\sigma_{1}=\left(\boldsymbol{r}_{1} \cdot \boldsymbol{v}_{1}\right) / \sqrt{\mu}$, and $r_{2}=\left\|f_{2} \boldsymbol{r}_{1}+g_{2} \boldsymbol{v}_{1}\right\|$. To evaluate the Lagrangian coefficients, the eccentric anomaly difference is computed by solving

$$
M_{2}-M_{1}=\left(E_{2}-E_{1}\right)+\frac{\sigma_{1}}{\sqrt{a}}\left[1-\cos \left(E_{2}-E_{1}\right)\right]-\left(1-\frac{r_{1}}{a}\right) \sin \left(E_{2}-E_{1}\right)
$$

where $M$ is the mean anomaly defined as $M=\sqrt{\mu / a^{3}}=n(t-\tau)$, in which $n$ is the mean motion and $\tau$ the time of passage through the perigee.

Typically (6) is solved with few Newton's iterations. Let us assume the dependence of the solution with respect to a set of parameters is of interest (e.g., with respect to the measurement errors as it appears later in Sect. IV, when the Kepler problem is solved within the IOD algorithm to obtain the O2S map). This dependence can be represented by a Taylor polynomial by applying the algorithm of Sec. II B. When the two-body problem approximation is adopted, the integration 
of Kepler's dynamics can be substituted by the solution of Kepler's problem, thus reducing the computational time.

\section{Lambert's problem}

In Lambert's problem, the initial position, final position, and the time of flight between the two positions are given. Solving Lambert's problem defines the Keplerian orbit that connects the two position vectors in the given time, allowing the calculation of the velocities at the initial and final positions.

Lambert's theorem states that the time of flight depends only on the semi-major axis of the connecting arc, the sum of the two radii $r_{1}+r_{2}$, and the distance between the initial and final positions, i.e., the chord length $c=\left\|\boldsymbol{r}_{2}-\boldsymbol{r}_{1}\right\|$ [36]. The two radii and the chord length are already known from the problem definition. The semi-major axis is the only unknown parameter, and it follows that it is possible to write the transfer time as a function of the semi-major axis only:

$$
\Delta t=f(a)
$$

all other variables in the equation should be known. This can be done as in [37], or the time of flight can be written as a function of some other parameters such as $p$ or $\Delta E$. In the approach adopted in this work, based on Battin's algorithm [15], the nonlinear equation to be solved is

$$
\log (A(x))-\log (\Delta t)=0
$$

in which

$$
A(x)=g(x)^{3 / 2}(\alpha(x)-\sin \alpha(x)-\beta(x)+\sin \beta(x)) .
$$

The functions $\alpha(x)$ and $\beta(x)$ are related to $x$ via the relations

$$
\sin ^{2} \frac{1}{2} \alpha(x)=\frac{s}{2 g(x)} \quad \sin ^{2} \frac{1}{2} \beta(x)=\frac{s-c}{2 g(x)},
$$

with

$$
g(x)=\frac{s}{2\left(1-x^{2}\right)}
$$


and the semi-perimeter

$$
s=\left(r_{1}+r_{2}+c\right) / 2
$$

The logarithm in (8) is adopted to facilitate the solution for $x$ by means of standard Newton's iteration. Note that the relation between $a$ and $x$ is simply given by

$$
a=\frac{s}{2\left(1-x^{2}\right)}
$$

Once the nominal solution is available, the dependence of the solution on an arbitrary set of parameters can be represented by a Taylor polynomial using the algorithm introduced in Section II B. The expansion of the solution of Lambert's problem with respect to observation uncertainties forms the basis of the IOD solver for both optical and radar observations, as described in Sec. IV A and IV B.

\section{Selection of the expansion order}

The selection of the expansion order is a crucial issue when using high order Taylor representation. The order must be selected so that the truncation error of the Taylor representation of the function of interest is lower than a threshold that is deemed appropriate for the problem at hand. In this work the function $f$ to be approximated is a generic $\mathcal{C}^{k+1}$ function that maps the input domain into the desired output space (e.g. the O2S map, which can include reference frame changes and propagations as explained in Sec. IV C and Sec. IV D). The input domain is typically scaled such that it is described by a $v$-dimensional hypercube $[-1,1]^{v}$. It is not possible to select a priori the expansion order $k$ to guarantee that the truncation error meets the accuracy requirements. However, once the Taylor approximation of order $k$ is computed, its truncation error can be estimated. Thus, one can either decide to work at an expansion order $k$ and then verify that the Taylor representation is accurate enough (the approach followed in this work) or can progressively increase the expansion order until the accuracy requirement is met. However, there are two limiting factors on the maximum order to be considered. The first one is that the computational time of a Taylor expansion increases exponentially with the expansion order and the problem dimension $v$. The second one is that the convergence radius of a Taylor series can be smaller than the uncertainty 
domain of interest. In both cases, the only option one can pursue is to split the initial domain in subdomains, which is the idea behind the technique of automatic domain splitting introduced in [38].

Three main methods exist for estimating the truncation error: a sample-based method, a coefficient-based method, and a rigorous estimation method. The sample-based method involves sampling the uncertainty domain and evaluating both the original function $f$ and its Taylor approximation $\mathcal{T}_{\boldsymbol{f}}$. The truncation error is readily estimated as the maximum difference between the value of the function and its Taylor approximation. The main drawback of this approach is that it can be time consuming and, by requiring the multiple evaluation of the $\boldsymbol{f}$, it can partially impair the advantage of working with Taylor approximations. However, as the truncation error increases with the distance from the Taylor expansion point, only few samples belonging to the boundary of the uncertainty domain (e.g. the corners of the $[-1,1]^{v}$ or just a subset of them in the direction of maximum error growth) can be used to estimated the truncation error.

The coefficient-based method has the advantage that it relies on the analysis of the coefficients of the Taylor approximation. Thus, it does not requires significant computational effort. The method estimates the size of the $k+1$ order terms of the Taylor polynomial based on an exponential fit of the size of all the known non-zero coefficients up to order $k$. The exponential fit is chosen because when the Taylor approximation is converging, the coefficients of the resulting polynomial expansion will in fact decay exponentially. This is a direct consequence of Taylor's Theorem. Given the $k$-th order Taylor representation of the function $f$

$$
\mathcal{T}_{\boldsymbol{f}}(\boldsymbol{x})=\sum_{\alpha} a_{\alpha} \boldsymbol{x}^{\alpha}
$$

written using multi-index notation, the size $S_{i}$ of the terms of order $i$ is computed as the sum of the absolute values of all coefficients of exact order $i$ :

$$
S_{i}=\sum_{|\alpha|=i}\left|a_{\alpha}\right| .
$$

We denote by $I$ the set of indices $i$ for which $S_{i}$ is non-zero. A least squares fit of the exponential function

$$
t(i)=A \cdot \exp (B \cdot i)
$$


is used to determine the coefficients $A, B$ such that $t(i)=S_{i}, i \in I$, is approximated optimally in the least squares sense. The value of $t(k+1)$ is used to estimate the size $S_{k+1}$ of the truncated order $k+1$ of $\mathcal{T}_{\boldsymbol{f}}$. This value is representative of the accuracy of the Taylor approximation of order $k$ in the domain of interest. Thus, for a given problem, the order $k$ to be used for the DA computations is selected so that $S_{k+1}$ is lower than a prescribed tolerance. It has to be remarked that this approach gives an accurate estimate of the truncation error when a sufficient number of coefficients is available for the fit, i.e. for a sufficiently high value of $k$ (say above 3). For more details the reader is referred to [38].

Lastly, the rigorous estimation method involves the use of interval algebra to rigorously bound the remainder term of the Taylor approximation, as implemented in the Taylor-model approach [39]. By being fully rigorous, this approach allows one to carry out validated computations, which can be used for example for computer assisted proofs. However, the use of this approach in complex engineering problems can require significant coding effort and can greatly increase the computational cost. For this reason the Taylor-model approach is not used in this work.

\section{Uncertainty mapping}

Consider two $n$-dimensional random vectors $\boldsymbol{x}$ and $\boldsymbol{y}$, related by the continuous one-to-one transformations $\boldsymbol{y}=\boldsymbol{f}(\boldsymbol{x})$ and $\boldsymbol{x}=\boldsymbol{g}(\boldsymbol{y})$, and the probability density function $p(\boldsymbol{x})$. The goal of uncertainty mapping is to represent the statistics of the transformed variable $\boldsymbol{y}$. This section first introduces a general method to map the pdf. The method relies on the high order Taylor expansion of the transformation functions $\boldsymbol{f}$ and $\boldsymbol{g}$, as well as of their Jacobians. Then, in Sec. IIIB an approach that exploits the availability of the Taylor representation of $\boldsymbol{f}$ to compute the $k$-th order approximation of the statistical moments of the mapped pdf is presented. Finally, a simpler method is introduced in Sec. III C, which exploits the same Taylor expansions to reduce the computational time of classical MC simulations.

\section{A. DA-based pdf mapping}

Using the axiom of probability conservation and the rules of change of variables in multiple integrals, the probability density function of $\boldsymbol{y}$ can be written as [28] 


$$
p(\boldsymbol{y})=p(\boldsymbol{g}(\boldsymbol{y}))\left|\operatorname{det} \frac{\partial \boldsymbol{g}}{\partial \boldsymbol{y}}\right|,
$$

in which det indicates the determinant. By substitution, Eq. (14) can be rewritten in terms of the original variables $\boldsymbol{x}$ as

$$
p(\boldsymbol{f}(\boldsymbol{x}))=p(\boldsymbol{x}) /\left|\operatorname{det} \frac{\partial \boldsymbol{f}}{\partial \boldsymbol{x}}\right| .
$$

The goal of the method is mapping the pdf of the observations into the pdf of the orbital parameters. Within this framework, the observations are represented by the vector $\boldsymbol{x}$ with known $p(\boldsymbol{x})$. As anticipated in Sec. I, there is no explicit relation between the observations and the orbital parameters in IOD. This means that the relation $\boldsymbol{y}=\boldsymbol{f}(\boldsymbol{x})$ is not known analytically and must be computed numerically by solving an implicit parametric relation in the form $\boldsymbol{h}(\boldsymbol{y}, \boldsymbol{x})=0$. This has always been the classical impediment to obtaining an analytical expression of the mapped pdf.

As explained in Section IIB, DA can solve the parametric implicit equation to provide a polynomial representation of $\boldsymbol{f}$, i.e. of the $\mathrm{O} 2 \mathrm{~S}$ map. The resulting polynomial can also be inverted to deliver the Taylor approximation of the S2O map

$$
\boldsymbol{x}=\mathcal{T}_{\boldsymbol{g}}(\boldsymbol{y})
$$

in which the superscript $k$ has been omitted for the sake of a lighter notation.

Furthermore, the Taylor expansion of the determinant of the Jacobian of both $\boldsymbol{f}$ and $\boldsymbol{g}$ can be easily obtained by carrying out the pertaining algebraic operations in the DA framework. Consequently, the computation of $p(\boldsymbol{y})$ can be obtained from Eq. (15) or Eq. (14) by exploiting the Taylor expansion of the determinants, i.e.

$$
p(\boldsymbol{y})=p\left(\mathcal{T}_{\boldsymbol{g}}(\boldsymbol{y})\right) \mathcal{T}_{\left|\operatorname{det} \frac{\partial \boldsymbol{g}}{\partial \boldsymbol{y}}\right|}(\boldsymbol{y})
$$

and

$$
p\left(\mathcal{T}_{\boldsymbol{f}}(\boldsymbol{x})\right)=p(\boldsymbol{x}) 1 / \mathcal{T}_{\left|\operatorname{det} \frac{\partial \boldsymbol{f}}{\partial \boldsymbol{x}}\right|}(\boldsymbol{x}) .
$$




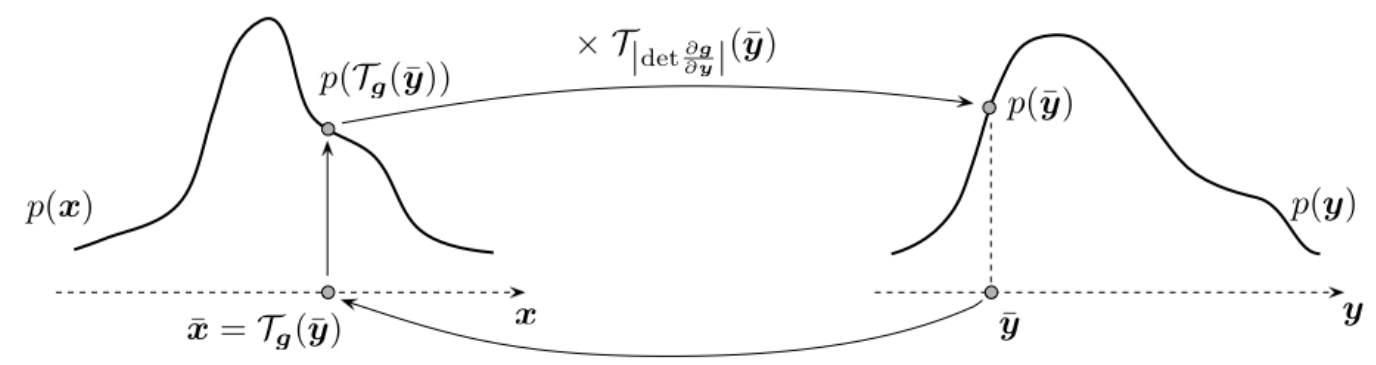

(a) Computation of $p(\boldsymbol{y})$ for a given $\overline{\boldsymbol{y}}$ using Eq. (17)

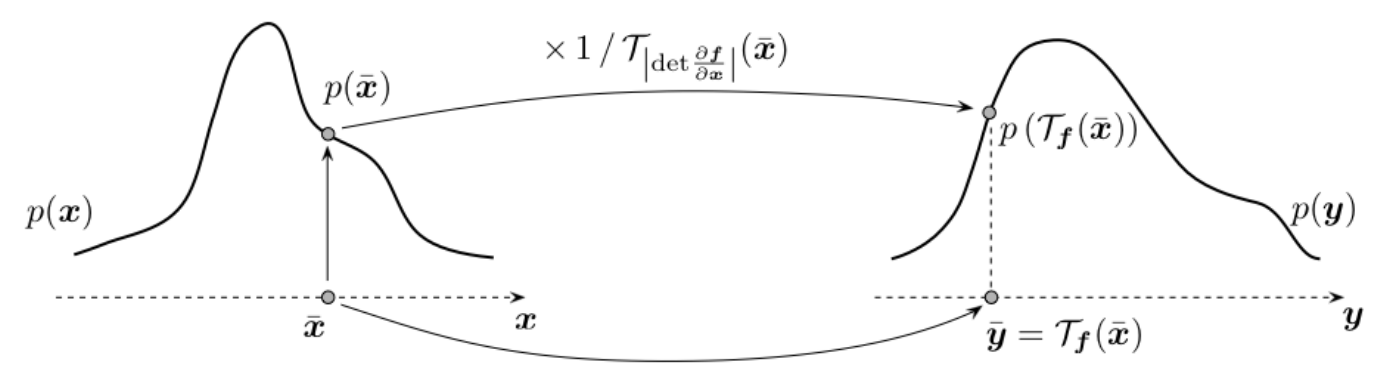

(b) Computation of $p\left(\mathcal{T}_{\boldsymbol{f}}(\boldsymbol{x})\right)$ for a given $\overline{\boldsymbol{x}}$ using Eq. (18)

Fig. 1: Schematic representation of the partial DA-based pdf mapping method.

In Eq. (17), for any given $\overline{\boldsymbol{y}}, p(\overline{\boldsymbol{y}})$ is computed by evaluating $\mathcal{T}_{\boldsymbol{g}}(\overline{\boldsymbol{y}})$ to obtain the corresponding $\overline{\boldsymbol{x}}$, computing $p(\overline{\boldsymbol{x}})$ from the known pdf of the observations, and multiplying the resulting pdf by the value of the Taylor expansion of the determinant of $\boldsymbol{g}$ at $\overline{\boldsymbol{y}}$ (see Fig. 1(a)). Alternatively, in Eq. (18), for any given $\overline{\boldsymbol{x}}$, the pdf at $\overline{\boldsymbol{y}}=\mathcal{T}_{\boldsymbol{f}}(\overline{\boldsymbol{x}})$ is computed by multiplying the corresponding $p(\overline{\boldsymbol{x}})$ by the inverse of the value of the Taylor expansion of the determinant of $\boldsymbol{f}$ at $\overline{\boldsymbol{x}}$ (see Fig. 1(b)).

This approach is labeled as partial DA-based pdf mapping method as, in both Eq. (17) and Eq. (18), the pdf of $\boldsymbol{y}$ is obtained by multiplying the value of the pdf of $\boldsymbol{x}$ by a Taylor polynomial. However, if $p(\boldsymbol{x})$ is known analytically, then both $p\left(\mathcal{T}_{\boldsymbol{g}}(\boldsymbol{y})\right)$ and $p(\boldsymbol{x})$ can be evaluated in the DA framework. The result is the Taylor expansion of $p(\boldsymbol{x})$ with respect to $\boldsymbol{y}$ and $\boldsymbol{x}$, respectively. Consequently, Eqs. (17) and (18) deliver a full polynomial representation of the mapped pdf, i.e.

$$
p(\boldsymbol{y}) \approx \mathcal{T}_{p(\boldsymbol{y})}(\boldsymbol{y})=\mathcal{T}_{p\left(\mathcal{T}_{\boldsymbol{g}}(\boldsymbol{y})\right)}(\boldsymbol{y}) \mathcal{T}_{\left|\operatorname{det} \frac{\partial \boldsymbol{g}}{\partial \boldsymbol{y}}\right|}(\boldsymbol{y})
$$


and

$$
p\left(\mathcal{T}_{\boldsymbol{f}}(\boldsymbol{x})\right) \approx \mathcal{T}_{p\left(\mathcal{T}_{\boldsymbol{f}}(\boldsymbol{x})\right)}(\boldsymbol{x})=\mathcal{T}_{p(\boldsymbol{x})}(\boldsymbol{x}) / \mathcal{T}_{\left|\operatorname{det} \frac{\partial \boldsymbol{f}}{\partial \boldsymbol{x}}\right|}(\boldsymbol{x})
$$

In Eq. (19), for any given $\overline{\boldsymbol{y}}, p(\overline{\boldsymbol{y}})$ is computed by evaluating directly $\mathcal{T}_{p(\boldsymbol{y})}(\boldsymbol{y})$ at $\overline{\boldsymbol{y}}$. Alternatively, in Eq. (20), for any given $\overline{\boldsymbol{x}}$, the pdf at $\overline{\boldsymbol{y}}=\mathcal{T}_{\boldsymbol{f}}(\overline{\boldsymbol{x}})$ is computed by evaluating directly $\mathcal{T}_{p\left(\mathcal{T}_{\boldsymbol{f}}(\boldsymbol{x})\right)}(\boldsymbol{x})$ at $\overline{\boldsymbol{x}}$. This second approach is referred to as full DA-based pdf mapping. Note that, since $p(\boldsymbol{x})$ is an asymptotic function, the accuracy of the Taylor expansions $\mathcal{T}_{p(\boldsymbol{y})}(\boldsymbol{y})$ and $\mathcal{T}_{p\left(\mathcal{T}_{\boldsymbol{f}}(\boldsymbol{x})\right)}(\boldsymbol{x})$ will be limited in this case, even when the computations are carried out at high orders.

\section{B. Computation of statistical moments}

The statistical moments of the mapped pdf can be computed in a deterministic way by computing the expectation of the analytical approximation of the O2S map $\mathcal{T}_{\boldsymbol{f}}(\boldsymbol{x})$, following the procedure already introduced in [27].

For a generic scalar random variable $x$ with pdf $p(x)$, the first four moments can be written as

$$
\left\{\begin{array}{l}
\mu=E\{x\} \\
P=E\left\{(x-\mu)^{2}\right\} \\
\gamma=\frac{E\left\{(x-\mu)^{3}\right\}}{\sigma^{3}} \\
\kappa=\frac{E\left\{(x-\mu)^{4}\right\}}{\sigma^{4}}-3,
\end{array}\right.
$$

where $\mu$ is the mean value, $P$ is the covariance, $\gamma$ and $\kappa$ are the skewness and the kurtosis, respectively, and the expectation value of $x$ is defined as

$$
E\{x\}=\int_{-\infty}^{+\infty} x p(x) d x
$$

So, the moments of the transformed pdf can be computed by applying the multivariate form of Eq. (21) to the Taylor expansion of the O2S map, $\mathcal{T}_{\boldsymbol{f}}(\boldsymbol{x})$. The result for the first two moments becomes

$$
\left\{\begin{array}{l}
\boldsymbol{\mu}_{i}=E\left\{\mathcal{T}_{\boldsymbol{f}}(\boldsymbol{x})_{i}\right\}=\sum_{p_{1}+\cdots+p_{n} \leq k} \boldsymbol{c}_{i, p_{1} \ldots p_{n}} E\left\{\delta x_{1}^{p_{1}} \cdots \delta x_{n}^{p_{n}}\right\} \\
\boldsymbol{P}_{i j}=E\left\{\left(\mathcal{T}_{\boldsymbol{f}}(\boldsymbol{x})_{i}-\boldsymbol{\mu}_{i}\right)\left(\mathcal{T}_{\boldsymbol{f}}(\boldsymbol{x})_{j}-\boldsymbol{\mu}_{j}\right)\right\}=\sum_{\substack{p_{1}+\cdots+p_{n} \leq k, q_{1}+\cdots+q_{n} \leq k}} \boldsymbol{c}_{i, p_{1} \ldots p_{n}} \boldsymbol{c}_{j, q_{1} \ldots q_{n}} E\left\{\delta x_{1}^{p_{1}+q_{1}} \cdots \delta x_{n}^{p_{n}+q_{n}}\right\},
\end{array}\right.
$$


where $\boldsymbol{c}_{i, p_{1} \ldots p_{n}}$ are the Taylor coefficients of the Taylor polynomial describing the $i$-th component of $\mathcal{T}_{\boldsymbol{f}}(\boldsymbol{x})$. Note that in the covariance matrix formula the coefficients $\boldsymbol{c}_{i, p_{1} \ldots p_{n}}$ and $\boldsymbol{c}_{j, q_{1} \ldots q_{n}}$ already include the subtraction of the mean terms. The coefficients of the higher order moments are computed by implementing the required operations on the Taylor polynomials in the DA framework. The expectation values on the right side of Eq. (23) are function of $p(\boldsymbol{x})\left(\right.$ e.g. $\left(\mathcal{T}_{\boldsymbol{f}}(\boldsymbol{x})_{i}-\boldsymbol{\mu}_{i}\right)\left(\mathcal{T}_{\boldsymbol{f}}(\boldsymbol{x})_{j}-\boldsymbol{\mu}_{j}\right)$ for the second order moment). It follows that, if the initial distribution is known, all of the moments of the transformed pdf $p(\boldsymbol{y})$ can be calculated. The number of monomials for which it is necessary to compute the expectation increases with the order of the Taylor expansion and, of course, with the order of the moment we want to compute. Note that, at this time, no hypothesis on the initial pdf has been made. Thus, the method can be applied independently of the considered variable distribution.

However, the Gaussian hypothesis is commonly used to represent the distribution of measurement noise. So, we can restrict to the case where $\boldsymbol{x}$ is a Gaussian random variable (GRV), $\boldsymbol{x} \sim \mathcal{N}(\boldsymbol{\mu}, \boldsymbol{P})$, in which $\boldsymbol{\mu}$ is the mean vector and $\boldsymbol{P}$ the covariance matrix. An important property of Gaussian distributions is that the statistics of a GRV can be completely described by the first two moments. In case of zero mean, the expression for computing higher-order moments in terms of the covariance matrix is due to Isserlis [40]. In physics literature, Isserlis's formula is known as the Wick's formula.

Let $s_{1}$ to $s_{n}$ be nonnegative integers, and $s=s_{1}+s_{2}+\cdots+s_{n}$. Then the Wick's formula suggests that

$$
E\left\{x_{1}^{s_{1}} x_{2}^{s_{2}} \ldots x_{n}^{s_{n}}\right\}= \begin{cases}0, & \text { if } s \text { is odd } \\ \operatorname{Haf}(\boldsymbol{P}), & \text { if } s \text { is even }\end{cases}
$$

where $\operatorname{Haf}(\boldsymbol{P})$ is the hafnian of $P=\left(\sigma_{i j}\right)$, which is defined as

$$
H a f(\boldsymbol{P})=\sum_{p \in \prod_{s}} \prod_{i=1}^{\frac{s}{2}} \sigma_{p_{2 i-1}, p_{2 i}},
$$

and $\prod_{s}$ is the set of all permutations $p$ of $\{1,2, \ldots, s\}$ satisfying the property $p_{1}<p_{3}<p_{5}<\ldots<$ $p_{s-1}$ and $p_{1}<p_{2}, p_{3}<p_{4}, \ldots, p_{s-1}<p_{s}[41]$.

We observe that the expectation value terms of Eq. (23) can be computed using Eq. (24), 
and the resulting moments can be used to describe the transformed pdf. As a result, if one is only interested in the statistical moments of the mapped pdf, MC simulations are not necessary, and this result represents an additional advantage of having an analytical approximation of the $\mathrm{O} 2 \mathrm{~S}$ function.

\section{DA-based Monte Carlo}

The DA-based MC method requires only the expansion of the $\boldsymbol{f}$ function, obtained as explained in Sec. IIB. The method is based on the fact that evaluating $\boldsymbol{y}=\mathcal{T}_{\boldsymbol{f}(\boldsymbol{x})}(\boldsymbol{x})$ is in general much simpler (and faster) than evaluating $\boldsymbol{y}=\boldsymbol{f}(\boldsymbol{x})$. On the other hand, if the expansion order is properly selected, the function $\boldsymbol{f}$ is sufficiently regular, and the uncertainty set of $\boldsymbol{x}$ is not too large, the error introduced by substituting $\boldsymbol{f}$ with its Taylor approximation is limited. Based on these considerations, the DA-based MC method is a classical MC method in which the repetitive evaluation of $\boldsymbol{f}$ is replaced by the evaluation of $\mathcal{T}_{\boldsymbol{f}}$ : given a set of samples of $\boldsymbol{x}$, the polynomial $\mathcal{T}_{\boldsymbol{f}}$ is evaluated repetitively and a statistical analysis is performed on the resulting $\boldsymbol{y}$. It is worth noting that, in the DA-based approach, the Taylor expansion $\mathcal{T}_{\boldsymbol{f}}$ shall be computed only once, since the same polynomial can be used in case new statistics need to be mapped. This is an additional advantage with respect to classical MC, as already pointed out in [42].

\section{Example: Cartesian to polar transformation}

The problem of mapping a Gaussian pdf from Cartesian to polar coordinates is investigated in this section for illustrative purposes. The section focuses on the full and partial DA-based pdf mapping methods, since the application of the MC method is deemed to be straightforward. The Cartesian to polar coordinate transformation is given by

$$
\boldsymbol{y}=\boldsymbol{f}(\boldsymbol{x})=\left(\begin{array}{c}
\sqrt{x_{1}^{2}+x_{2}^{2}} \\
\operatorname{atan} \frac{x_{2}}{x_{1}}
\end{array}\right)
$$

and $\boldsymbol{x}$ is assumed to be a Gaussian random vector (GRV), with $p(\boldsymbol{x})=\mathcal{N}(\boldsymbol{\mu}, \boldsymbol{P})$, in which $\boldsymbol{\mu}=\left(\mu_{1}, \mu_{2}\right)$ is the mean vector and $\boldsymbol{P}$ is a diagonal covariance matrix defined by the standard deviations $\sigma_{1}$ and $\sigma_{2}$. The goal is to compute the mapped pdf $p(\boldsymbol{y})$. 
The analytical solution is available in this simple case. To write Eq. (14), the inverse transformation is

$$
\boldsymbol{x}=\boldsymbol{g}(\boldsymbol{y})=\left(\begin{array}{l}
y_{1} \cos y_{2} \\
y_{1} \sin y_{2}
\end{array}\right)
$$

and the determinant of its Jacobian

$$
\left|\operatorname{det} \frac{\partial \boldsymbol{g}}{\partial \boldsymbol{y}}\right|=y_{1}
$$

Thus, based on Eq. (14), the mapped pdf can be written as

$$
p(\boldsymbol{y})=\mathcal{N}(\boldsymbol{\mu}, \boldsymbol{P}) y_{1},
$$

which yields

$$
p(\boldsymbol{y})=\frac{\exp \left(-\frac{\left(y_{1} \cos \left(y_{2}\right)-\mu_{1}\right)^{2}}{2 \sigma_{1}^{2}}-\frac{\left(y_{1} \sin \left(y_{2}\right)-\mu_{2}\right)^{2}}{2 \sigma_{2}^{2}}\right) y_{1}}{2 \pi \sigma_{1} \sigma_{2}} .
$$

Thus, $p(\boldsymbol{y})$ is obtained explicitly in terms $\boldsymbol{y}$ : for any $\overline{\boldsymbol{y}}$, the evaluation of Eq. (30) supplies $p(\boldsymbol{y})$ at $\bar{y}$.

The derivation of the inverse transformation of Eq. (27) can be avoided using Eq. (15), which in this case reads

$$
p(\boldsymbol{y})=p(\boldsymbol{f}(\boldsymbol{x}))=\mathcal{N}(\boldsymbol{\mu}, \boldsymbol{P}) \sqrt{x_{1}^{2}+x_{2}^{2}}
$$

which makes use of the relation

$$
1 /\left|\operatorname{det} \frac{\partial \boldsymbol{f}}{\partial \boldsymbol{x}}\right|=\sqrt{x_{1}^{2}+x_{2}^{2}}
$$

In its explicitly form, Eq. (31) reads

$$
p(\boldsymbol{y})=p(\boldsymbol{f}(\boldsymbol{x}))=\frac{\exp \left(-\frac{\left(x_{1}-\mu_{1}\right)^{2}}{2 \sigma_{1}^{2}}-\frac{\left(x_{2}-\mu_{2}\right)^{2}}{2 \sigma_{2}^{2}}\right) \sqrt{x_{1}^{2}+x_{2}^{2}}}{2 \pi \sigma_{1} \sigma_{2}} .
$$

In this case, $p(\boldsymbol{y})$ is obtained in terms of $\boldsymbol{x}$ : for any $\overline{\boldsymbol{x}}$, the evaluation of Eq. (33) supplies $p(\boldsymbol{y})$ at $\overline{\boldsymbol{y}}=\boldsymbol{f}(\overline{\boldsymbol{x}})$.

Figure 2(a) shows the contour levels of $p(\boldsymbol{x})$ for $\boldsymbol{\mu}=(5,4)$ and $\boldsymbol{\sigma}=(1,1)$, in the range $\boldsymbol{x} \in$ $\boldsymbol{\mu} \pm \frac{3}{2} \boldsymbol{\sigma}$. In Figure 2(b), the contour levels of $p(\boldsymbol{y})$ are plotted using the analytical expression (30) or (33) equally. 


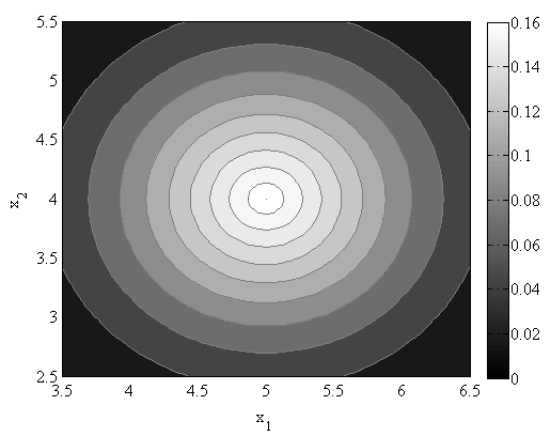

(a) Initial pdf in cartesian variables

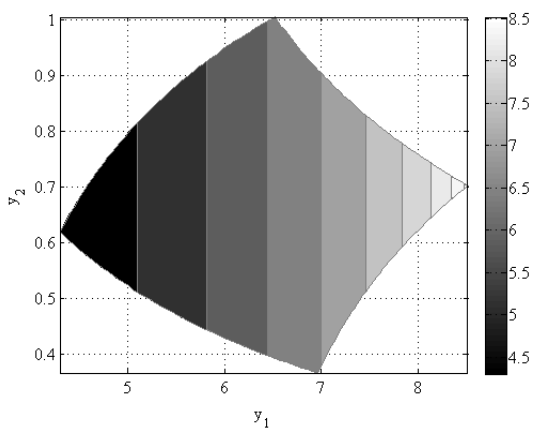

(c) Contour levels of $1 / \mathcal{T}_{\left|\operatorname{det} \frac{\partial \boldsymbol{f}}{\partial \boldsymbol{x}}\right|}(\boldsymbol{x})$

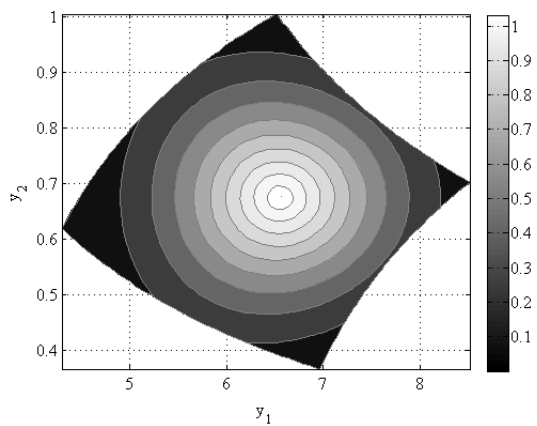

(e) Mapped pdf with the partial DA method

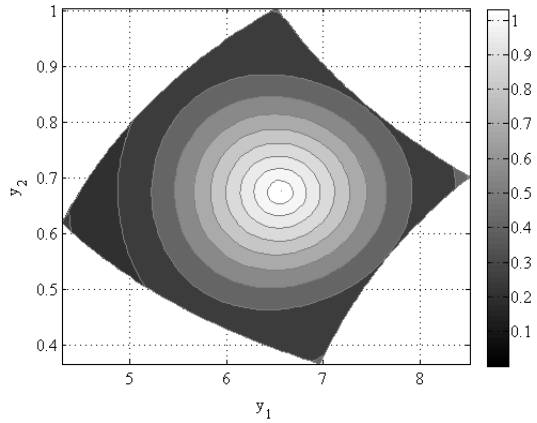

(g) Mapped pdf with the full DA method

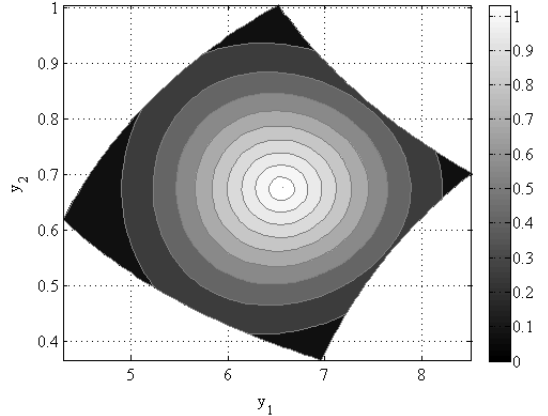

(b) Mapped pdf in polar variables

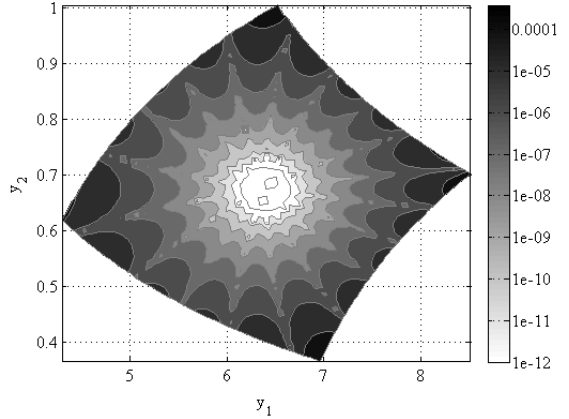

(d) Absolute error of $1 / \mathcal{T}_{\left|\operatorname{det} \frac{\partial \boldsymbol{f}}{\partial \boldsymbol{x}}\right|}(\boldsymbol{x})$

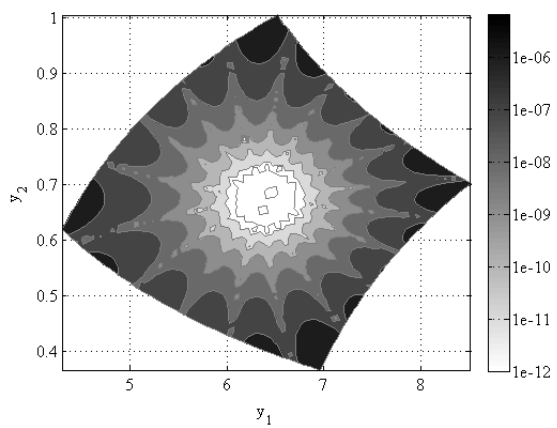

(f) Absolute error of the partial DA method

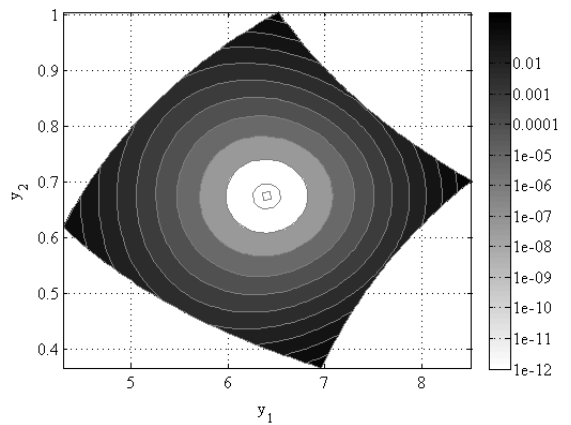

(h) Absolute error of the full DA method

Fig. 2: Pdf mapping for the Cartesian to polar transformation: analytic solutions vs. Taylor approximation. 
Figures 2(c) to 2(h) assess the accuracy of both the partial and full DA-based pdf mapping methods using the particle-based method introduced in Sec. II C on a very fine grid for visualisation purposes. The approach of Fig. 1(b) is adopted for the partial method. The vector $\boldsymbol{x}$ is initialized as DA variable to compute the Taylor approximation of Eqs. (26) and (32) in the DA framework. Then, for each $\boldsymbol{x} \in \boldsymbol{\mu} \pm \frac{3}{2} \boldsymbol{\sigma}$, the resulting Taylor polynomials are evaluated to compute the approximated values of both $\boldsymbol{y}$ and the inverse of the determinant of the Jacobian. The contour levels of $1 / \mathcal{T}_{\mid \operatorname{det} \frac{\partial \boldsymbol{f}}{\partial \boldsymbol{x} \mid}}(\boldsymbol{x})$ are plotted in Figure 2(c), whereas Figure 2(d) shows the absolute error of its 9-th order Taylor approximation with respect to the analytical solution. The maximum absolute error of the Taylor representation in the considered region is $3.84 \times 10^{-4}$. Once the Taylor expansion of the inverse of the determinant of the Jacobian is available, its multiplication by $p(\boldsymbol{x})$ supplies the mapped pdf $p(\boldsymbol{y})$. The result is shown in Figure 2(e). Clearly, the absolute error of the Taylor approximation of $p(\boldsymbol{y})$ with respect to the analytical solution, reported in Figure 2(f), has the same behaviour of that of the Taylor representation of the inverse of the determinant of the Jacobian. This is due to the fact that $p(\boldsymbol{y})$ is obtained by multiplication with $p(\boldsymbol{x})$, which is imposed and, then, known analytically. The maximum absolute error of the partial DA method in the considered region is $6.64 \times 10^{-6}$.

Figure 2(g) shows the contour levels of the mapped pdf obtained with the full DA method, i.e. when also $p(\boldsymbol{x})$ is approximated as a Taylor polynomial. The error of the mapped pdf in this case is a combination of the error of the Taylor approximations of both $p(\boldsymbol{x})$ and the inverse of the determinant of the Jacobian. However, Figure 2(h) clearly shows that the error on the polynomial representation of $p(\boldsymbol{x})$ prevails: the absolute error is $4.67 \times 10^{-1}$, resulting in a maximum percentage error of $327.62 \%$. The relative error becomes large when the pdf gets small because the Taylor expansion fails to represent the asymptotic behaviour of $p(\boldsymbol{x})$ independently of the order used. Of course, the error would increase if a larger initial domain is considered, e.g. for $\boldsymbol{x} \in \boldsymbol{\mu} \pm 3 \boldsymbol{\sigma}$. In this case, considering that the error is mainly due to the approximation of $p(\boldsymbol{x})$ which is axisymmetric, the truncation error of the full DA approach can be estimated by comparing the value of $p(\boldsymbol{x})$ and its DA approximation at any point at which the Mahalanobis distance is 3 . In this example, $p(\boldsymbol{\mu}+3 \boldsymbol{\sigma})=1.7681 \times 10^{-3}$, whereas $\mathcal{T}_{p}(\boldsymbol{\mu}+3 \boldsymbol{\sigma})=1.3565$. This result clearly shows 
the intrinsic unsuitability of the DA approach when approximating an asymptotic function with a single expansion. A single polynomial expansion is not sufficient to represent accurately $p(\boldsymbol{x})$ and, consequently, $p(\boldsymbol{y})$ over the entire domain.

\section{Probabilistic IOD}

\section{A. Angles-only IOD}

In the classical angles-only IOD problem, three optical observations at epoch $t_{i}$, with $i=1, \ldots, 3$ are available. The observations consist of three couples of right ascension and declination angles $\left(\alpha_{i}, \delta_{i}\right)$, or in a more compact notation $(\boldsymbol{\alpha}, \boldsymbol{\delta})$. These observations provide three inertial lines of sight $\hat{\boldsymbol{\rho}}_{i}$, i.e. the unit vectors pointing from the observer (on the Earth's surface) to the observed object.

A method to use Taylor DA to solve and expand the solution of the corresponding IOD problem has already been introduced by Armellin et al. [43]. This considers all the slant ranges $\rho_{i}$ with $i=1, \ldots, 3$ (in a more compact notation $\boldsymbol{\rho}$ ) as the unknowns of the problem, for which initial guesses are provided by a classical Gauss' method. Starting from these initial guesses, two Lambert's problem, a first one between $t_{1}$ and $t_{2}$ and a second one between $t_{2}$ and $t_{3}$, can be solved to obtain the two velocity vectors $\boldsymbol{v}_{2}^{-}$and $\boldsymbol{v}_{2}^{+}$. These two velocity vectors will be initially different as only first guesses of the slant ranges are available. Thus, the IOD problem consists in finding the $\boldsymbol{\rho}$ such that the residual function $\boldsymbol{h}(\boldsymbol{\rho})=\boldsymbol{v}_{2}^{-}-\boldsymbol{v}_{2}^{+}=0$. Typically just few Newton iterations are sufficient to find the $\boldsymbol{\rho}$ that solves this set of three nonlinear equations [44]. Once $\boldsymbol{\rho}$ is known, the position vectors $\boldsymbol{r}_{i}$ can be readily computed; the associated velocity vectors are delivered by solving the two Lambert problems. If the measurement angles are defined as DA numbers, the residuals become functions of both the slant ranges and measurements angles, i.e. $\boldsymbol{v}_{2}^{-}-\boldsymbol{v}_{2}^{+}=\boldsymbol{h}(\boldsymbol{\rho}, \boldsymbol{\alpha}, \boldsymbol{\delta})$. The goal is to find $\boldsymbol{\rho}=\mathcal{T}_{\boldsymbol{\rho}}(\boldsymbol{\alpha}, \boldsymbol{\delta})$, i.e. a Taylor approximation of the function that tells how the slant ranges need to change to solve the IOD problem for any variation of the measurements within their uncertainty domain. This is exactly the problem described in Sec. IIB. When $\mathcal{T}_{\boldsymbol{\rho}}(\boldsymbol{\alpha}, \boldsymbol{\delta})$ is computed, the $\mathcal{T}_{\boldsymbol{r}_{i}}(\boldsymbol{\alpha}, \boldsymbol{\delta})$ are readily available. Finally, the $\mathcal{T}_{\boldsymbol{v}_{i}}(\boldsymbol{\alpha}, \boldsymbol{\delta})$ are obtained by solving the Lambert problems in the DA framework. The polynomials $\mathcal{T}_{\boldsymbol{r}_{i}}$ and $\mathcal{T}_{\boldsymbol{v}_{i}}$ can then be concatenated to 
write the full state of the spacecraft at the epoch of the second observation as a Taylor polynomial $\boldsymbol{y}_{2}=\mathcal{T}_{\boldsymbol{y}_{2}}(\boldsymbol{\alpha}, \boldsymbol{\delta})$.

For any value of $\boldsymbol{\alpha}$ and $\boldsymbol{\delta}$ belonging to their uncertainty domain, the evaluation of the polynomial $\mathcal{T}_{\boldsymbol{y}_{2}}$ supplies a Taylor approximation of the state that solves the IOD problem. Evidently, this Taylor polynomial is the approximation of the O2S map for the angles-only IOD, which supplies the estimated spacecraft state for different values of the measurements. If the pdf of the observations, i.e. $p(\boldsymbol{\alpha}, \boldsymbol{\delta})$, is known, the DA-based pdf mapping methods can be used to estimate the spacecraft state pdf at $t_{2}$. If the initial pdf is assumed to be Gaussian, the method described in Sec. IIIB can be used to compute the statistical moments of the mapped pdf.

\section{B. Two position vectors IOD}

In the case of radar observations, two measurements at epoch $t_{i}$, with $i=1,2$, are available. Each observation consists of the pointing angles $\left(\alpha_{i}, \delta_{i}\right)$ and the range $\rho_{i}$, or in a more compact form $(\boldsymbol{\alpha}, \boldsymbol{\delta}, \boldsymbol{\rho})$. By assuming the observer's location as known, each radar observation is equivalent to measuring the spacecraft position vector. This greatly simplifies the IOD problem. If all $\alpha_{i}, \delta_{i}$, and $\rho_{i}$ are initialized as DA numbers, simple algebraic operations allows the two position vectors to be approximated in terms of their Taylor expansion with respect to the measurements, i.e. as $\boldsymbol{r}_{i}=\mathcal{T}_{\boldsymbol{r}_{i}}\left(\rho_{i}, \alpha_{i}, \delta_{i}\right)$.

A Lambert problem is formulated with the resulting two position vectors and the elapsed time between the observations. The Lambert problem can be solved in the DA framework as summarised in Sec. II B 2 [45]. The result is the Taylor approximation of the velocity vectors at the two observation epochs, i.e. $\boldsymbol{v}_{i}=\mathcal{T}_{\boldsymbol{v}_{i}}(\boldsymbol{\alpha}, \boldsymbol{\delta}, \boldsymbol{\rho})$, for $i=1,2$. The polynomials $\mathcal{T}_{\boldsymbol{r}_{i}}$ and $\mathcal{T}_{\boldsymbol{v}_{i}}$ can then be concatenated to write the full state of the spacecraft at the epoch of the first observation as a Taylor polynomial $\boldsymbol{y}_{1}=\mathcal{T}_{\boldsymbol{y}_{1}}(\boldsymbol{\alpha}, \boldsymbol{\delta}, \boldsymbol{\rho})$.

The polynomial $\mathcal{T}_{\boldsymbol{y}_{1}}$ is the Taylor expansion of the solution of the IOD problem with respect to the measurements: the evaluation of the polynomial $\mathcal{T}_{\boldsymbol{y}_{1}}$ for any $\boldsymbol{\alpha}, \boldsymbol{\delta}$, and $\boldsymbol{\rho}$ delivers the state $\boldsymbol{y}_{1}$ that solves the problem. Clearly, $\mathcal{T}_{\boldsymbol{y}_{1}}$ is the approximation of the O2S map for the two position vectors IOD. Similarly to Sec. IV A, if the pdf of the observations, $p(\boldsymbol{\alpha}, \boldsymbol{\delta}, \boldsymbol{\rho})$, is known, the DA- 
based pdf mapping methods can be used to approximate the pdf of the estimated state at $t_{1}$. If the initial pdf is assumed to be Gaussian, the method described in Sec. III B can be used to compute the statistical moments of the mapped pdf.

\section{IOD in high-fidelity dynamics}

The procedures for the angles-only IOD and the two position vectors IOD presented in the previous sections rely on the assumption that the object moves in Keplerian dynamics. However, both procedures can be extended to work for arbitrary dynamics. This section addresses such a generalization, starting from the procedure for the angles-only IOD and then moving to the case of the two position vectors.

Let us assume that a reference state $\overline{\boldsymbol{y}}_{2}$ has been estimated in Keplerian dynamics from the reference optical observations $\left(\bar{\alpha}_{i}, \bar{\delta}_{i}\right)$ at epoch $t_{i}$, with $i=1, \ldots, 3$, or, with the compact notation, from $(\overline{\boldsymbol{\alpha}}, \overline{\boldsymbol{\delta}})$. The generalization of the angles-only procedure goes through the following steps:

1. The state at epoch $t_{2}$ and all the measurements are initialized as DA numbers, i.e. $\boldsymbol{y}_{2}, \boldsymbol{\alpha}$, and $\delta$, around their reference values.

2. Using simple algebraic operations, the measurements at $t_{2}$ are computed from the state $\boldsymbol{y}_{2}$ to obtain the polynomials $\alpha_{2}^{c}=\mathcal{T}_{\alpha_{2}^{c}}\left(\boldsymbol{y}_{2}\right)$ and $\delta_{2}^{c}=\mathcal{T}_{\delta_{2}^{c}}\left(\boldsymbol{y}_{2}\right)$.

3. The state vector $\boldsymbol{y}_{2}$ is propagated forward to $t_{3}$ and backward to $t_{1}$ in the dynamics of interest using the DA version of the 7/8 Dormand-Prince integrator described in Sec. II A. The propagations yield the Taylor expansions of the state vector at $t_{1}$ and $t_{3}$ with respect to $\boldsymbol{y}_{2}$, i.e. $\boldsymbol{y}_{1}=\mathcal{T}_{\boldsymbol{y}_{1}}\left(\boldsymbol{y}_{2}\right)$ and $\boldsymbol{y}_{3}=\mathcal{T}_{\boldsymbol{y}_{3}}\left(\boldsymbol{y}_{2}\right)$.

4. Similarly to step 2, the measurements at $t_{1}$ and $t_{3}$ are computed from $\boldsymbol{y}_{1}$ and $\boldsymbol{y}_{3}$, respectively, to obtain the polynomials $\alpha_{i}^{c}=\mathcal{T}_{\alpha_{i}^{c}}\left(\boldsymbol{y}_{2}\right)$ and $\delta_{i}^{c}=\mathcal{T}_{\delta_{i}^{c}}^{c}\left(\boldsymbol{y}_{2}\right)$, with $i=1$ and 3. Consequently, the measurements at all epochs have been computed in terms of their Taylor expansion in $\boldsymbol{y}_{2}$. With a compact notation, these polynomials are denoted as $\left(\boldsymbol{\alpha}^{c}, \boldsymbol{\delta}^{c}\right)=\mathcal{T}_{\boldsymbol{\alpha}^{c}, \boldsymbol{\delta}^{c}}\left(\boldsymbol{y}_{2}\right)$.

5. Clearly, the computed $\boldsymbol{\alpha}^{c}$ and $\boldsymbol{\delta}^{c}$ must match $\boldsymbol{\alpha}$ and $\boldsymbol{\delta}$. To impose this condition, the errors $\Delta \boldsymbol{\alpha}$ and $\Delta \boldsymbol{\delta}$ between $\left(\boldsymbol{\alpha}^{c}, \boldsymbol{\delta}^{c}\right)$ and $(\boldsymbol{\alpha}, \boldsymbol{\delta})$ are computed. By definition, these errors depend on 
all $\boldsymbol{y}_{2}, \boldsymbol{\alpha}$, and $\boldsymbol{\delta}$ :

$$
(\Delta \boldsymbol{\alpha}, \Delta \boldsymbol{\delta})=\left(\boldsymbol{\alpha}^{c}-\boldsymbol{\alpha}, \boldsymbol{\delta}^{c}-\boldsymbol{\delta}\right)=\left(\mathcal{T}_{\boldsymbol{\alpha}^{c}}\left(\boldsymbol{y}_{2}\right)-\boldsymbol{\alpha}, \mathcal{T}_{\boldsymbol{\delta}^{c}}\left(\boldsymbol{y}_{2}\right)-\boldsymbol{\delta}\right)=\mathcal{T}_{\Delta \boldsymbol{\alpha}, \Delta \boldsymbol{\delta}}\left(\boldsymbol{y}_{2}, \boldsymbol{\alpha}, \boldsymbol{\delta}\right)
$$

6. The polynomial in Eq. (34) can be inverted using the dedicated algorithm available in the DA framework [25] to obtain:

$$
\boldsymbol{y}_{2}=\mathcal{T}_{\boldsymbol{y}_{2}}(\Delta \boldsymbol{\alpha}, \Delta \boldsymbol{\delta}, \boldsymbol{\alpha}, \boldsymbol{\delta})
$$

7. The condition $(\Delta \boldsymbol{\alpha}, \Delta \boldsymbol{\delta})=(0,0)$ can now be imposed to obtain

$$
\boldsymbol{y}_{2}=\mathcal{T}_{\left.\boldsymbol{y}_{2}\right|_{\Delta \boldsymbol{\alpha}, \Delta \delta=0}}(\boldsymbol{\alpha}, \boldsymbol{\delta})
$$

which is the Taylor expansion of the estimated state at $t_{2}$ with respect to the measurements $(\boldsymbol{\alpha}, \boldsymbol{\delta})$ at $t_{i}$, with $i=1, \ldots, 3$.

The evaluation of the polynomial map in Eq. (36) at any $(\boldsymbol{\alpha}, \boldsymbol{\delta})$ delivers the corresponding estimated state $\boldsymbol{y}_{2}$ in the dynamics used in step 3. Consequently, it is the Taylor expansion of the O2S map in the dynamics of interest. Similarly to the previous sections, the pdf of the observations, $p(\boldsymbol{\alpha}, \boldsymbol{\delta})$, can be mapped into $\boldsymbol{y}_{2}$ by applying the DA-based pdf mapping methods on $\mathcal{T}_{\left.\boldsymbol{y}_{2}\right|_{\Delta \boldsymbol{\alpha}, \Delta \delta=0}}$.

The above algorithm can be adapted to the two position vectors IOD. In this case, the reference state $\overline{\boldsymbol{y}}_{1}$ is obtained by solving the Lambert problem in the Keplerian dynamics between the position vectors associated to the reference measurements $\left(\bar{\alpha}_{i}, \bar{\delta}_{i}, \bar{\rho}_{i}\right)$ at epoch $t_{i}$, with $i=1,2$, or, with the compact notation, $(\overline{\boldsymbol{\alpha}}, \overline{\boldsymbol{\delta}}, \overline{\boldsymbol{\rho}})$. The generalization of the procedure of Sec. IV B for arbitrary dynamics goes through the following steps:

1. The state at epoch $t_{1}$ and all the measurements are initialized as DA numbers, i.e. $\boldsymbol{y}_{1}, \boldsymbol{\alpha}, \boldsymbol{\delta}$, and $\boldsymbol{\rho}$, around their reference values.

2. Using simple algebraic operations, the measurements at $t_{1}$ are computed from the state $\boldsymbol{y}_{1}$ in the DA framework: $\left(\alpha_{1}^{c}, \delta_{1}^{c}, \rho_{1}^{c}\right)=\mathcal{T}_{\alpha_{1}^{c}, \delta_{1}^{c}, \rho_{1}^{c}}\left(\boldsymbol{y}_{1}\right)$.

3. The state vector $\boldsymbol{y}_{1}$ is propagated forward to the epoch $t_{2}$ of the second observation in the dynamics of interest. Once again, the propagation is carried out with the DA version of the 
7/8 Dormand-Prince integrator. The propagation yields the Taylor expansion of the state vector at $t_{2}$ with respect to $\boldsymbol{y}_{1}$, i.e. $\boldsymbol{y}_{2}=\mathcal{T}_{\boldsymbol{y}_{2}}\left(\boldsymbol{y}_{1}\right)$.

4. The measurements at $t_{2}$ are computed from $\boldsymbol{y}_{2}$ to obtain the polynomials $\left(\alpha_{2}^{c}, \delta_{2}^{c}, \rho_{2}^{c}\right)=$ $\mathcal{T}_{\alpha_{2}^{c}, \delta_{2}^{c}, \rho_{2}^{c}}\left(\boldsymbol{y}_{1}\right)$. All the measurements have now been computed in terms of their Taylor expansions in $\boldsymbol{y}_{1}$, i.e. $\left(\boldsymbol{\alpha}^{c}, \boldsymbol{\delta}^{c}, \boldsymbol{\rho}^{c}\right)=\mathcal{T}_{\boldsymbol{\alpha}^{c}, \boldsymbol{\delta}^{c}, \boldsymbol{\rho}^{c}}\left(\boldsymbol{y}_{1}\right)$.

5. The computed $\left(\boldsymbol{\alpha}^{c}, \boldsymbol{\delta}^{c}, \boldsymbol{\rho}^{c}\right)$ must match $(\boldsymbol{\alpha}, \boldsymbol{\delta}, \boldsymbol{\rho})$. To impose this condition, the error between the observables is obtained as

$$
(\Delta \boldsymbol{\alpha}, \Delta \boldsymbol{\delta}, \Delta \boldsymbol{\rho})=\left(\boldsymbol{\alpha}^{c}-\boldsymbol{\alpha}, \boldsymbol{\delta}^{c}-\boldsymbol{\delta}, \boldsymbol{\rho}^{c}-\boldsymbol{\rho}\right)=\mathcal{T}_{\Delta \boldsymbol{\alpha}, \Delta \boldsymbol{\beta}, \Delta \boldsymbol{\rho}}\left(\boldsymbol{y}_{1}, \boldsymbol{\alpha}, \boldsymbol{\delta}, \boldsymbol{\rho}\right)
$$

6. The polynomial in Eq. (37) is inverted to obtain:

$$
\boldsymbol{y}_{1}=\mathcal{T}_{\boldsymbol{y}_{1}}(\Delta \boldsymbol{\alpha}, \Delta \boldsymbol{\delta}, \Delta \boldsymbol{\rho}, \boldsymbol{\alpha}, \boldsymbol{\delta}, \boldsymbol{\rho})
$$

7. The condition $(\Delta \boldsymbol{\alpha}, \Delta \boldsymbol{\delta}, \Delta \boldsymbol{\rho})=(0,0,0)$ can now be imposed to obtain

$$
\boldsymbol{y}_{1}=\mathcal{T}_{\left.\boldsymbol{y}_{1}\right|_{\Delta \alpha, \Delta \delta, \Delta \rho=0}}(\boldsymbol{\alpha}, \boldsymbol{\delta}, \boldsymbol{\rho})
$$

Similarly to Eq. (36), the polynomial map in Eq. (39) is the Taylor expansion of the estimated state at $t_{1}$ with respect to the measurements $(\boldsymbol{\alpha}, \boldsymbol{\delta}, \boldsymbol{\rho})$ at $t_{1}$ and $t_{2}$, i.e. the O2S map of the two position vector IOD problem. The map has been obtained in the general dynamics adopted in Step 3. Once the pdf of the measurements is available, this polynomial can be used to map it into $\boldsymbol{y}_{1}$ using the DA-based pdf mapping methods.

\section{Arbitrary state representations}

As detailed in the previous section, once the polynomial map of Eq. (36) or the one of Eq. (39) is available, the DA-based pdf mapping methods described in Sec. III A can be used to map the pdf of the measurements into the pdf of the estimated states. This is achieved by setting the function $\boldsymbol{f}$ of Eq. (15) to $\mathcal{T}_{\left.\boldsymbol{y}_{2}\right|_{\Delta \boldsymbol{\alpha}, \Delta \delta=0}}(\boldsymbol{\alpha}, \boldsymbol{\delta})$ or $\mathcal{T}_{\left.\boldsymbol{y}_{1}\right|_{\Delta \boldsymbol{\alpha}, \Delta \delta, \Delta \rho=0}}(\boldsymbol{\alpha}, \boldsymbol{\delta}, \boldsymbol{\rho})$, respectively. Indeed, they provide the transformation $\boldsymbol{y}=\boldsymbol{f}(\boldsymbol{x})$ from the observations $\boldsymbol{x}$ to the state vector $\boldsymbol{y}$ in terms of its Taylor expansion $\boldsymbol{y}=\mathcal{T}_{\boldsymbol{f}}(\boldsymbol{x})$. 
Without loss of generality, let us assume that the above transformation has been derived by representing the state vector $\boldsymbol{y}$ in a set of orbital parameters $O P 1$. Thus, $\boldsymbol{y}_{O P 1}=\boldsymbol{f}_{O P 1}(\boldsymbol{x})$ and the DA-based solution of the IOD problem has delivered its Taylor expansion $\boldsymbol{y}_{O P 1}=\mathcal{T}_{\boldsymbol{f}_{O P 1}}(\boldsymbol{x})$, which is valid from the measurements $\boldsymbol{x}$ to the $O P 1$ representation of $\boldsymbol{y}$. It is worth observing that the DA-based pdf mapping method works for $\boldsymbol{y}$ expressed in any set of orbital parameters, provided that the necessary coordinate transformations are included in the function $f$. The coordinate transformation from $O P 1$ to any new set of orbital parameters $O P 2$ is usually given by a nonlinear function $\boldsymbol{y}_{O P 2}=\boldsymbol{f}_{O P 1 \rightarrow O P 2}\left(\boldsymbol{y}_{O P 1}\right)$, which consists of algebraic operations. The transformation $\boldsymbol{f}_{O P 1}$ can be composed with $\boldsymbol{f}_{O P 1 \rightarrow O P 2}$ to obtain the overall transformation

$$
\boldsymbol{y}_{O P 2}=\boldsymbol{f}_{O P 2}(\boldsymbol{x})=\boldsymbol{f}_{O P 1 \rightarrow O P 2} \circ \boldsymbol{f}_{O P 1}(\boldsymbol{x})
$$

from the observations $\boldsymbol{x}$ to the $O P 2$ representation of $\boldsymbol{y}$. The evaluation of Eq. (40) in the DA framework provides its Taylor expansion

$$
\boldsymbol{y}_{O P 2}=\mathcal{T}_{\boldsymbol{f}_{O P 2}}(\boldsymbol{x})=\mathcal{T}_{\boldsymbol{f}_{O P 1 \rightarrow O P 2}} \circ \mathcal{T}_{\boldsymbol{f}_{O P 1}}(\boldsymbol{x}),
$$

where $\mathcal{T}_{f_{O P 1 \rightarrow O P 2}}$ is the Taylor expansion of the coordinate transformation from $O P 1$ to $O P 2$. Consequently, the DA-based pdf mapping methods can be applied on $\mathcal{T}_{f_{O P 2}}$ to map the pdf of the observations, $p(\boldsymbol{x})$, into the pdf of the estimated state $\boldsymbol{y}$ represented in the arbitrary set of orbital parameters $O P 2$.

\section{Test Cases}

Single-pass optical and radar observations of the two objects listed in Table 1 are considered as test cases for the DA-based pdf mapping methods. The first one is INTELSAT 901, a GEO communications spacecraft. The second one is an SL-8 rocket body in LEO. 
Table 1: Test cases: orbital parameters

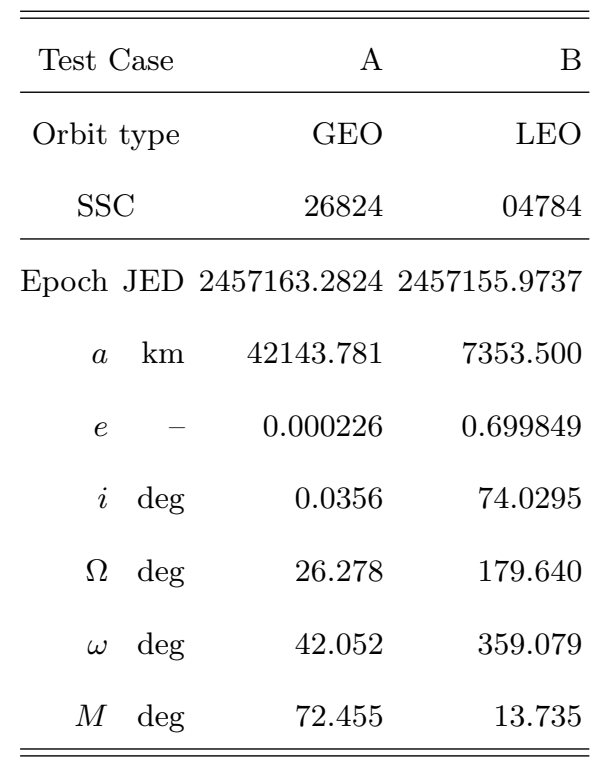

For the object in GEO, an optical campaign is simulated with three observations taken with the European Space Agency Optical Ground Station (OGS, Teide Observatory, Canary Islands). The observations are separated by $12 \mathrm{~min}$ (approximately by an arc length of $3 \mathrm{deg}$ ). A resolution of $\sigma_{\alpha, \delta}=0.5$ arcsec is considered for both the right ascension and declination.

For the LEO case, two radar observations taken with the Chilbolton Advanced Meteorological Radar (CAMRa, Chilbolton Observatory, UK) are simulated. The observations are separated by 2 minutes, approximately an arc length of $7 \mathrm{deg}$, such that the range from the station remains of the order of $1000 \mathrm{~km}$. The range has a resolution of $\sigma_{\rho}=25 \mathrm{~m}$, and the pointing angles have standard deviations $\sigma_{\alpha, \delta}=0.25$ deg.

In both campaigns, the observations are simulated using the following procedure. First of all, a set of measurements is generated using the orbital parameters of Table 1, without adding any noise. As solving the IOD problem with these measurements would deliver the true solutions (i.e. the orbital parameters of Table 1), these measurements are referred to as true measurements. Then, the simulated optical and radar observations are generated by adding a random error to the true measurements, using the standard deviations introduced above. The resulting observations are labelled as nominal as they are the reference observations in the IOD solution processes described 
in Sec. IV; i.e. all the Taylor expansions are computed around them. The nominal observations are a particular realization of the noisy measurements, and thus differ from the true observations. Accordingly, the nominal solution of the IOD process will differ from its true solution. To highlight this difference the nominal and the true solutions will be indicated in the analysis of the results with a diamond and a square marker, respectively. Unless differently specified, the results were obtained using Taylor expansions at 6 -th order. This value was selected based on the accuracy analyses reported in the discussion of the results.

\section{A. Test Case A}

Figure 3 shows the results of the 6-th dimensional pdf mapped from the observation space into the modified equinoctial elements space $\boldsymbol{y}_{M E E}=(p, f, g, h, k, L)$, in which $p=a\left(1-e^{2}\right)$ is the semilatus rectum, $f=e \cos (\omega+\Omega)$ and $g=e \sin (\omega+\Omega), h=\tan (i / 2) \cos (\Omega)$ and $k=\tan (i / 2) \sin (\Omega)$, and the true longitude $L=\Omega+\omega+\nu$. The set $\boldsymbol{y}_{C O E}=(a, e, i, \Omega, \omega, \nu)$ represents instead the classical orbital elements. The two-body dynamics is used in this case. The plots are obtained by processing 100,000 normally distributed vectors generated in the observation space using the MATLAB function mvnrnd. Each sample is mapped from the observation space $(\boldsymbol{\alpha}, \boldsymbol{\delta})$ into the state space $\boldsymbol{y}_{M E E}$ at the epoch of the second observation by evaluating the Taylor approximation $\boldsymbol{y}_{M E E}=\mathcal{T}_{\boldsymbol{y}_{M E E}}(\boldsymbol{\alpha}, \boldsymbol{\delta})$, i.e. the straightforward application of the DA-based MC method described in Sec. III C.

As explained in Sec. III A, the pdf of each observation sample is obtained using the partial DAbased pdf mapping method, i.e. by multiplying the pdf $p(\boldsymbol{\alpha}, \boldsymbol{\delta})$ of the measurements by the Taylor representation of the inverse of the determinant of the Jacobian of the O2S map, i.e. $p\left(\boldsymbol{y}_{M E E}\right)=$ $p\left(\mathcal{T}_{\boldsymbol{y}_{M E E}}(\boldsymbol{\alpha}, \boldsymbol{\delta})\right)=p(\boldsymbol{\alpha}, \boldsymbol{\delta}) 1 /\left|\mathcal{T}_{\frac{\partial \boldsymbol{y}_{M E E}}{\partial(\boldsymbol{\alpha}, \boldsymbol{\delta})}}\right|$. The colour of each sample is based on the value assumed by the pdf, which has been scaled in the range $[0,1]$ for a better readability of the plots.

The figures on the left column of Fig. 3 show the projection of the pdf on the $(p, L),(f, g)$, and $(h, k)$ planes. In these plots, the true (square marker) and the nominal (diamond marker) IOD solutions are reported. As anticipated at the beginning of Sec. V, the true solution differs from the nominal solution of the probabilistic IOD, since the nominal observations are a particular realization of the noisy measurements. On the other hand, as expected, the true solution belongs 
to the uncertainty set resulting from the probabilistic IOD process.

It is worth mentioning that, unlike an MC simulation, in which one needs to evaluate the O2S map in many samples to be able to perform a meaningful statistical analysis of the results, the samples are here used only for visualisation purposes. In fact, by using the partial DA-based approach, the pdf of each sample can be directly computed by evaluating the associated analytical functions, without resorting to a statistical analysis performed on a set of samples. 


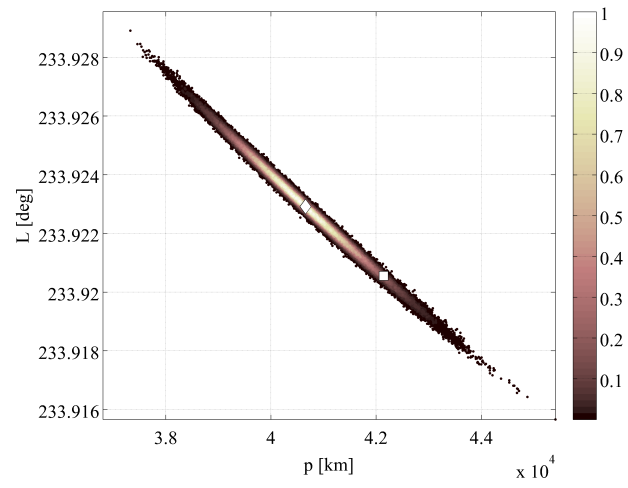

(a)

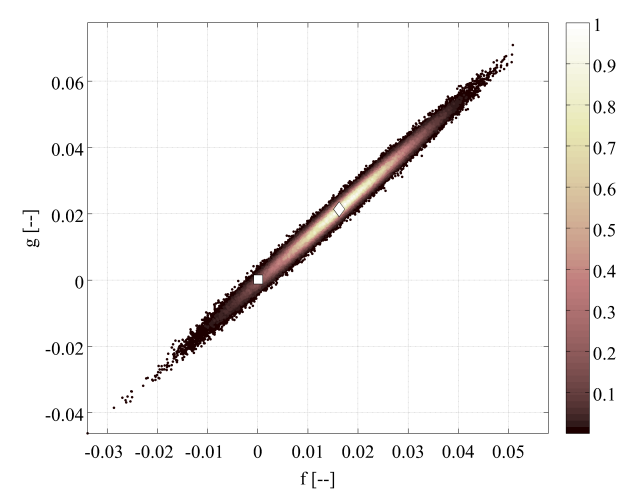

(c)

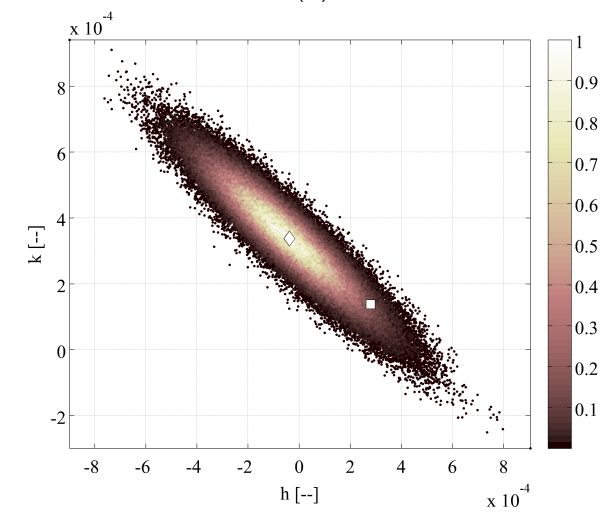

(e)

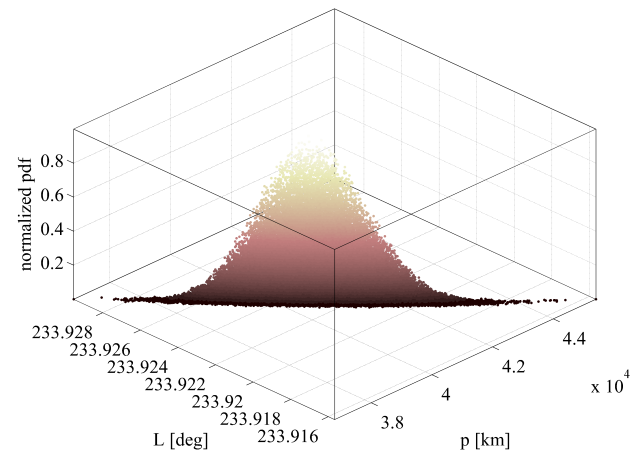

(b)

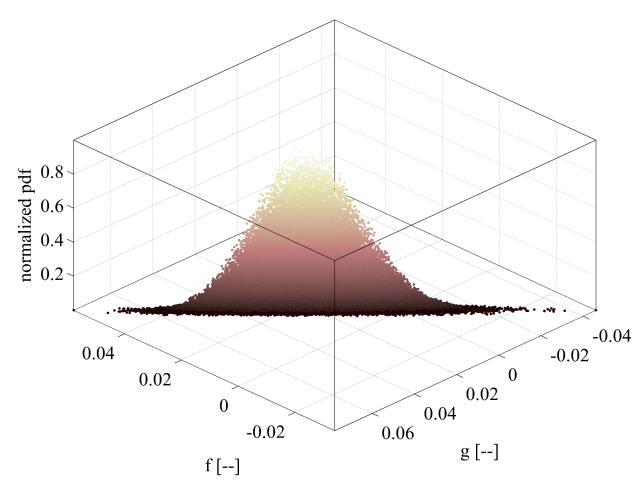

(d)

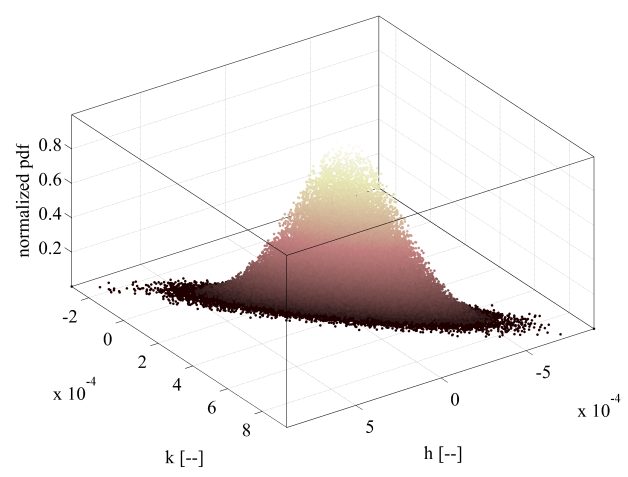

(f)

Fig. 3: Mapped pdf in the MEE space for optical observations

Figure 3 shows that, although the size of the uncertainty map is large, the mapped pdf still (visually) resembles a Gaussian distribution. The uncertainty set is large as the optical observations are taken on a short arc, and, thus, the sensitivity of the solution to the measurements is high. In addition, in a short arc, the nonlinearities of the dynamics do not play a significant role and, 
thus, the pdf remains nearly Gaussian. This qualitative argument is confirmed by comparing the covariance-correlation matrices computed at 6-th and 1-st order, which are reported in Table 2 and 3 respectively. The difference between the two matrices in all the components is less than $1 \%$. These matrices were computed with the approach described in Sec. III B, i.e. without using samples.

\begin{tabular}{cccccc}
\hline \hline $\mathrm{p}[\mathrm{km}]$ & $\mathrm{f}$ & $\mathrm{g}$ & $\mathrm{h}$ & $\mathrm{k}$ & $\mathrm{L}[\mathrm{rad}]$ \\
\hline $7.9707 \mathrm{e}+05$ & $-8.4471 \mathrm{e}+00$ & $-1.1551 \mathrm{e}+01$ & $1.6564 \mathrm{e}-01$ & $-1.1906 \mathrm{e}-01$ & $-2.3042 \mathrm{e}-02$ \\
$-9.9701 \mathrm{e}-01$ & $9.0058 \mathrm{e}-05$ & $1.2202 \mathrm{e}-04$ & $-1.7723 \mathrm{e}-06$ & $1.2389 \mathrm{e}-06$ & $2.4297 \mathrm{e}-07$ \\
$-9.9913 \mathrm{e}-01$ & $9.9297 \mathrm{e}-01$ & $1.6768 \mathrm{e}-04$ & $-2.3883 \mathrm{e}-06$ & $1.7422 \mathrm{e}-06$ & $3.3485 \mathrm{e}-07$ \\
$9.9128 \mathrm{e}-01$ & $-9.9783 \mathrm{e}-01$ & $-9.8543 \mathrm{e}-01$ & $3.5029 \mathrm{e}-08$ & $-2.3938 \mathrm{e}-08$ & $-4.7516 \mathrm{e}-09$ \\
$-9.6999 \mathrm{e}-01$ & $9.4962 \mathrm{e}-01$ & $9.7864 \mathrm{e}-01$ & $-9.3033 \mathrm{e}-01$ & $1.8901 \mathrm{e}-08$ & $3.4957 \mathrm{e}-09$ \\
$-9.9659 \mathrm{e}-01$ & $9.8867 \mathrm{e}-01$ & $9.9850 \mathrm{e}-01$ & $-9.8033 \mathrm{e}-01$ & $9.8184 \mathrm{e}-01$ & $6.7066 \mathrm{e}-10$ \\
\hline \hline
\end{tabular}

Table 2: Covariance values between states (upper triangle), correlation values (lower triangle), and variances (diagonal terms) computed at 6-th order for optical observations.

\begin{tabular}{cccccc}
\hline \hline $\mathrm{p}[\mathrm{km}]$ & $\mathrm{f}$ & $\mathrm{g}$ & $\mathrm{h}$ & $\mathrm{k}$ & $\mathrm{L}[\mathrm{rad}]$ \\
\hline $7.9373 \mathrm{e}+05$ & $-8.4153 \mathrm{e}+00$ & $-1.1508 \mathrm{e}+01$ & $1.6525 \mathrm{e}-01$ & $-1.1877 \mathrm{e}-01$ & $-2.2987 \mathrm{e}-02$ \\
$-9.9701 \mathrm{e}-01$ & $8.9756 \mathrm{e}-05$ & $1.2161 \mathrm{e}-04$ & $-1.7687 \mathrm{e}-06$ & $1.2364 \mathrm{e}-06$ & $2.4248 \mathrm{e}-07$ \\
$-9.9914 \mathrm{e}-01$ & $9.9295 \mathrm{e}-01$ & $1.6712 \mathrm{e}-04$ & $-2.3835 \mathrm{e}-06$ & $1.7387 \mathrm{e}-06$ & $3.3417 \mathrm{e}-07$ \\
$9.9146 \mathrm{e}-01$ & $-9.9795 \mathrm{e}-01$ & $-9.8553 \mathrm{e}-01$ & $3.4998 \mathrm{e}-08$ & $-2.3915 \mathrm{e}-08$ & $-4.7472 \mathrm{e}-09$ \\
$-9.7014 \mathrm{e}-01$ & $9.4966 \mathrm{e}-01$ & $9.7874 \mathrm{e}-01$ & $-9.3026 \mathrm{e}-01$ & $1.8884 \mathrm{e}-08$ & $3.4925 \mathrm{e}-09$ \\
$-9.9678 \mathrm{e}-01$ & $9.8877 \mathrm{e}-01$ & $9.9862 \mathrm{e}-01$ & $-9.8032 \mathrm{e}-01$ & $9.8182 \mathrm{e}-01$ & $6.7005 \mathrm{e}-10$ \\
\hline \hline
\end{tabular}

Table 3: Covariance values between states (upper triangle), correlation values (lower triangle), and variances (diagonal terms) computed at 1-st order for optical observations.

It is important to notice that the selection of MEE is particularly suited to maintain the normality of the statistics. The effect of selecting different orbital elements representations on the mapped pdf is shown in Fig. 4(a), in which the dimensionality of the pdf is reduced to 2 by binning in the semi-major axis - eccentricity space. Since the orbital eccentricity cannot take negative values, the mapped pdf is clearly visually non Gaussian when classical orbital elements are used. This is fur- 
ther highlighted by the normality plots in Fig. 5. In particular, Figure 5(f) shows that also the true anomaly is not normally distributed. This is due to both the low eccentricity and low inclination of the test orbit: a small variation in the observations can produce a large variation in all the true anomaly, the pericenter anomaly and the right ascension of the node. The true longitude (the sum of these three angles) is significantly less affected.

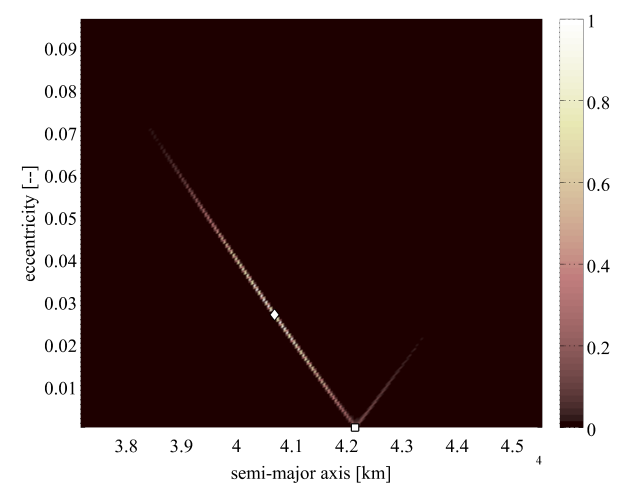

(a) Samples binned in the semi-major axis - eccentricity space

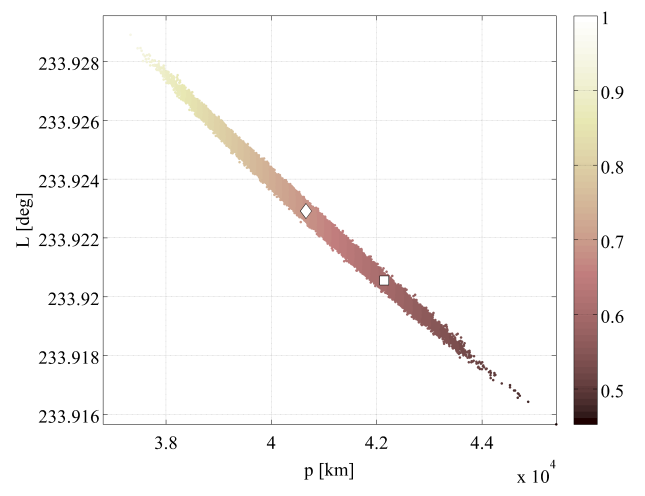

(b) Mapped pdf in the semilatus rectum - true longitude space for uniform distribution

Fig. 4: Analysis of the impact of state representation and initial pdf on the mapped pdf for optical observations. 


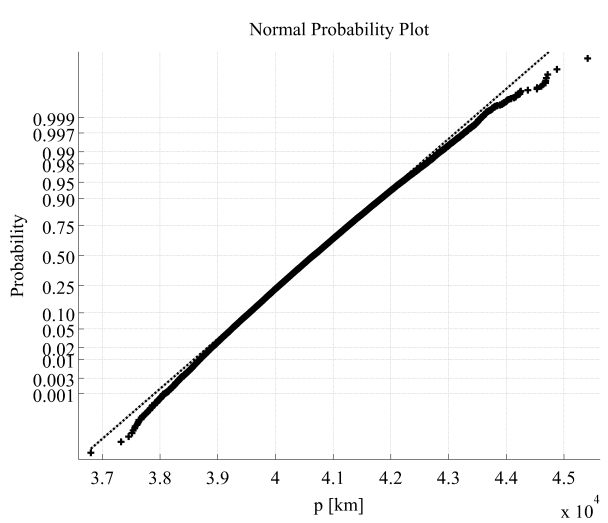

(a)

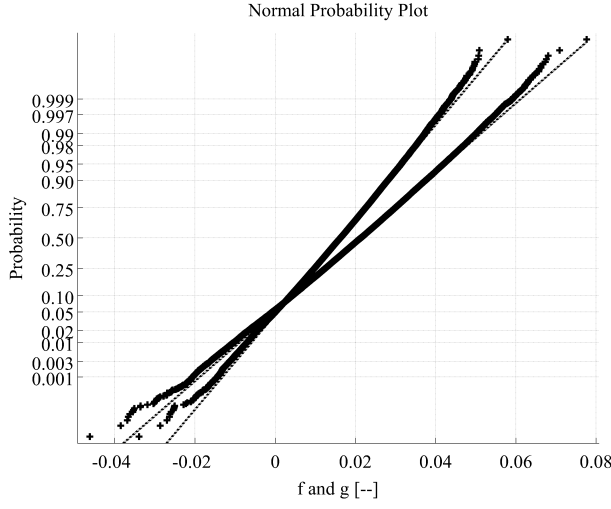

(c)

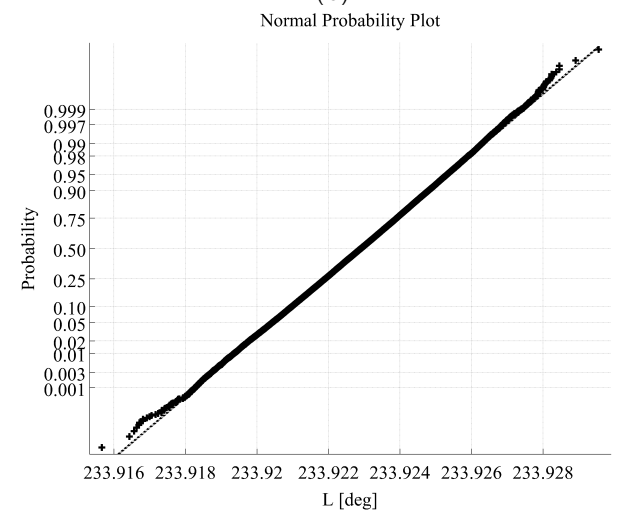

(e)

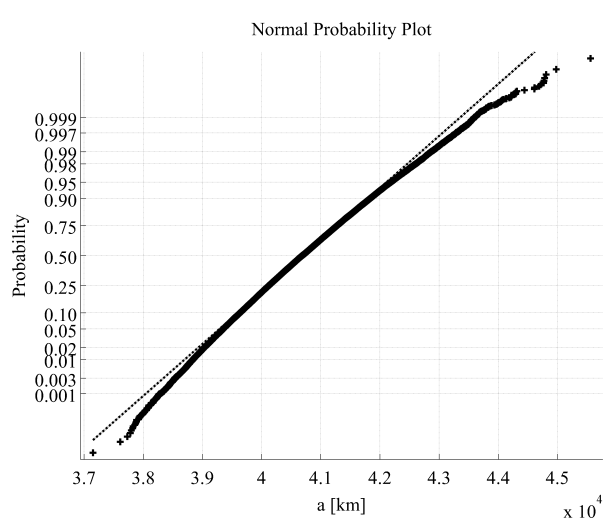

(b)

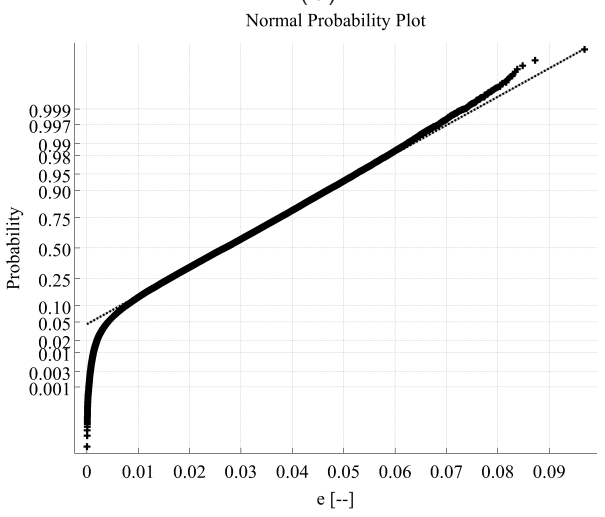

(d)

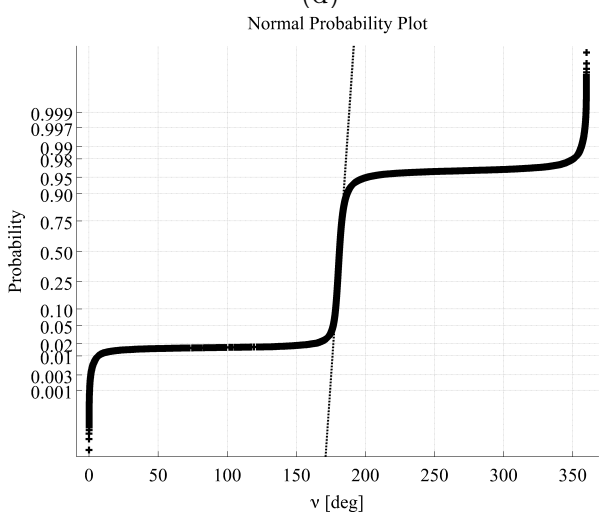

(f)

Fig. 5: Normality plots for optical observations: comparison between MEE (left) and COE (right). The dashed lines are extensions of the line connecting the 25th and 75th percentiles of the distribution: proximity of the distributions to the dashed lines indicates validity of the normality assumption.

The proposed DA-based pdf mapping method is independent of the particular pdf adopted for 
the observations. Figure 4(b) illustrates the result of mapping a pdf that is uniformly distributed in the 6 -th dimensional cube of side $\left[-3 \sigma_{\boldsymbol{\alpha}, \boldsymbol{\delta}}, 3 \sigma_{\boldsymbol{\alpha}, \boldsymbol{\delta}}\right]$ in the observation space. In this particular case, the mapped pdf depends only on the determinant of the Jacobian of the transformation, since $p(\boldsymbol{\alpha}, \boldsymbol{\delta})$ is constant over the entire cube.

The proposed mapping method can be used to map a pdf to any arbitrary time. To this aim, suppose the Taylor expansion of the O2S map has been obtained at the epoch of the second observation $t_{2}$ using the techniques introduced in Sec. IV:

$$
\boldsymbol{y}_{2}=\mathcal{T}_{\boldsymbol{y}_{2}}(\boldsymbol{\alpha}, \boldsymbol{\delta})
$$

The initial condition $\boldsymbol{y}_{2}$ can be propagated forward to any future epoch $t_{f}$ using the DA solution of the Kepler problem when two-body dynamics is considerd or the DA version of the 7/8 DormandPrince integrator for an arbitrary dynamical model. In either case the result is the Taylor expansion of the final state $\boldsymbol{y}_{f}$ with respect to the measurements $\boldsymbol{\alpha}$ and $\boldsymbol{\delta}$ taken at $t_{2}$, i.e.

$$
\boldsymbol{y}_{f}=\mathcal{T}_{\boldsymbol{y}_{f}}(\boldsymbol{\alpha}, \boldsymbol{\delta})
$$

It is worth observing that the polynomial map $\mathcal{T}_{\boldsymbol{y}_{f}}$ includes the dependencies on $\boldsymbol{\alpha}$ and $\boldsymbol{\delta}$ deriving from the solution of the IOD problem. Thus, given any specific set of observations $\overline{\boldsymbol{\alpha}}$ and $\overline{\boldsymbol{\delta}}$, the evaluation of Eq. (43) is an approximation of the result one would obtain by solving the IOD problem at $t_{0}$ and propagating the estimated state to $t_{f}$ in cascade. Similarly to Eq. (36) and Eq. (39), the polynomial $\mathcal{T}_{\boldsymbol{y}_{f}}$ can be used to map $p(\boldsymbol{\alpha}, \boldsymbol{\delta})$ into the pdf of $\boldsymbol{y}_{f}$ using the DA-based pdf mapping methods. To show the results of this procedure, Fig. 10 reports the 100,000 normally distributed samples introduced above after binning them in the space of the semilatus rectum and true longitude. On the left side the bins are plotted at the epoch of the second observation $t_{2}$, whereas on the right side a 6 -th order expansion of the solution of Kepler's problem is used to map them ahead for 1 day. It is apparent how the dynamics stretches the set along the orbit. After approximately one revolution the set is spread on an arc length of more than 50 deg. This result is supported also by the covariance-correlation matrix reported in Table 4, which shows an increase of four orders of magnitude in the $\sigma_{L}$ with respect to Table 2. Note that, as two body dynamics is assumed, this matrix correctly differs from the one at $t_{2}$ in the last column and row only. 


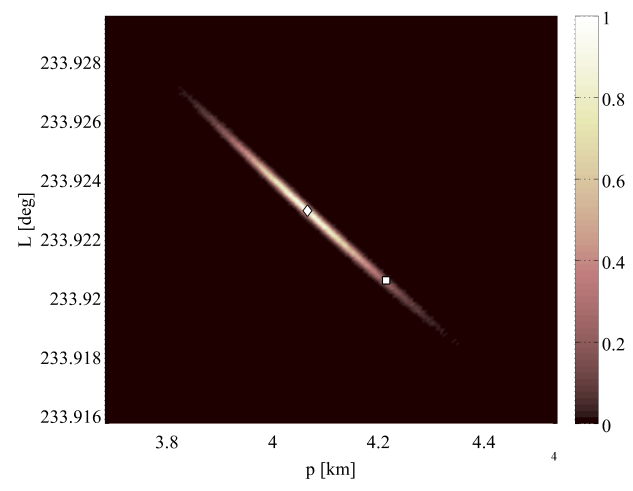

(a) Epoch $t_{2}$

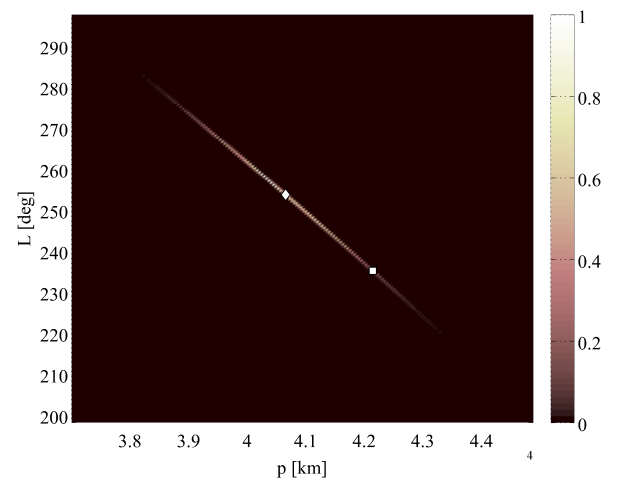

(b) Epoch $t_{2}+1$ day

Fig. 6: Samples binned in the semilatus rectum - true longitude space for optical observations at different epochs.

\begin{tabular}{cccccc}
\hline \hline $\mathrm{p}[\mathrm{km}]$ & $\mathrm{f}$ & $\mathrm{g}$ & $\mathrm{h}$ & $\mathrm{k}$ & $\mathrm{L}[\mathrm{rad}]$ \\
\hline $7.9707 \mathrm{e}+05$ & $-8.4471 \mathrm{e}+00$ & $-1.1551 \mathrm{e}+01$ & $1.6564 \mathrm{e}-01$ & $-1.1906 \mathrm{e}-01$ & $-1.7035 \mathrm{e}+02$ \\
$-9.9701 \mathrm{e}-01$ & $9.0058 \mathrm{e}-05$ & $1.2202 \mathrm{e}-04$ & $-1.7723 \mathrm{e}-06$ & $1.2389 \mathrm{e}-06$ & $1.8053 \mathrm{e}-03$ \\
$-9.9913 \mathrm{e}-01$ & $9.9297 \mathrm{e}-01$ & $1.6768 \mathrm{e}-04$ & $-2.3883 \mathrm{e}-06$ & $1.7422 \mathrm{e}-06$ & $2.4685 \mathrm{e}-03$ \\
$9.9128 \mathrm{e}-01$ & $-9.9783 \mathrm{e}-01$ & $-9.8543 \mathrm{e}-01$ & $3.5029 \mathrm{e}-08$ & $-2.3938 \mathrm{e}-08$ & $-3.5391 \mathrm{e}-05$ \\
$-9.6999 \mathrm{e}-01$ & $9.4962 \mathrm{e}-01$ & $9.7864 \mathrm{e}-01$ & $-9.3033 \mathrm{e}-01$ & $1.8901 \mathrm{e}-08$ & $2.5433 \mathrm{e}-05$ \\
$-9.9983 \mathrm{e}-01$ & $9.9683 \mathrm{e}-01$ & $9.9887 \mathrm{e}-01$ & $-9.9085 \mathrm{e}-01$ & $9.6935 \mathrm{e}-01$ & $3.6420 \mathrm{e}-02$ \\
\hline \hline
\end{tabular}

Table 4: Covariance values between states (upper triangle), correlation values (lower triangle), and variances (diagonal terms) computed at 6-th order after one revolution for optical observations.

The proposed methods are valid as long as the Taylor approximation of the transformations is accurate enough. The coefficient-based method presented in Sec. IIC was first used to verify that the truncation error of the 6-th order approximation of the O2S function was sufficiently small in the domain of interest. The results of this analysis are reported in Fig. 7(a), showing that the absolute errors are sufficiently small in the 6 -th dimensional cube of side $\left[-3 \sigma_{\boldsymbol{\alpha}, \boldsymbol{\delta}}, 3 \sigma_{\boldsymbol{\alpha}, \boldsymbol{\delta}}\right]$. However, in an IOD problem, it is more important to verify whether the predicted observations computed from the Taylor approximation of the state $\mathcal{T}_{\boldsymbol{y}_{2}}(\boldsymbol{\alpha}, \boldsymbol{\delta})$ are close enough to the real observations, for any admissible 
value of the measurements noise. In other words, we need to verify that the observational residuals derived from the evaluation of the Taylor approximation of the $\mathrm{O} 2 \mathrm{~S}$ function are sufficiently small. To verify this, samples are generated in the observation space (referred to as sampled observations) and the associated IOD solution is computed using the polynomial map. Then, each IOD solution is used to generate the predicted observations by evaluating the measurement function. The accuracy of the expansion is assessed by a statistical analysis of the difference between the sampled and the predicted observations, relative to the given perturbation; i.e. the relative residuals. This is a sample-based approach for checking the accuracy of the expansion (according to the classification proposed in Sec. II C), but it has the advantage of not requiring multiple pointwise solutions of the IOD problem, which would be time consuming. In this approach the accuracy analysis is focused on the observational residuals, which is what matters in the IOD problem: any state with sufficiently small residuals shall be regarded as a solution of the IOD problem. Figure 7(b) shows the empirical cumulative distribution function of the relative residuals for different expansion orders. The relative error decreases with the expansion order and a 6-th order computation guarantees a maximum relative error of the order of $1 \times 10^{-3}$, corresponding to maximum residual of $2.0771 \times 10^{-3} \operatorname{arcsec}$ $\left(1.0070 \times 10^{-8} \mathrm{rad}\right)$. This analysis clearly shows that the 6-th order expansion $\mathcal{T}_{\boldsymbol{y}_{2}}(\boldsymbol{\alpha}, \boldsymbol{\delta})$ can be regarded as an accurate approximation of the O2S function in the domain of interest.

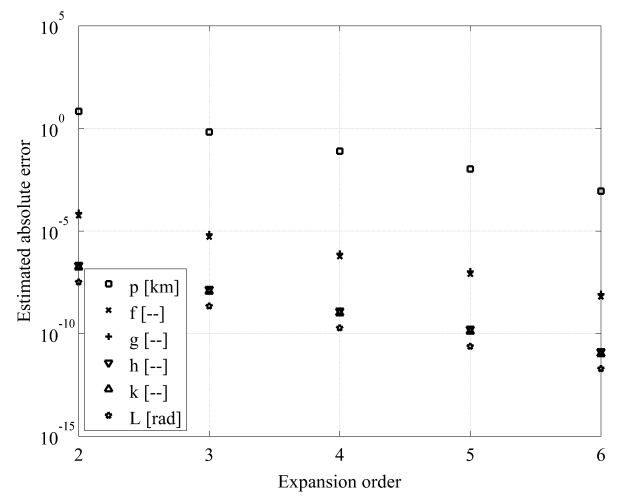

(a) Estimation of the truncation error of $\mathcal{T}_{\boldsymbol{y}_{2}}(\boldsymbol{\alpha}, \boldsymbol{\delta})$

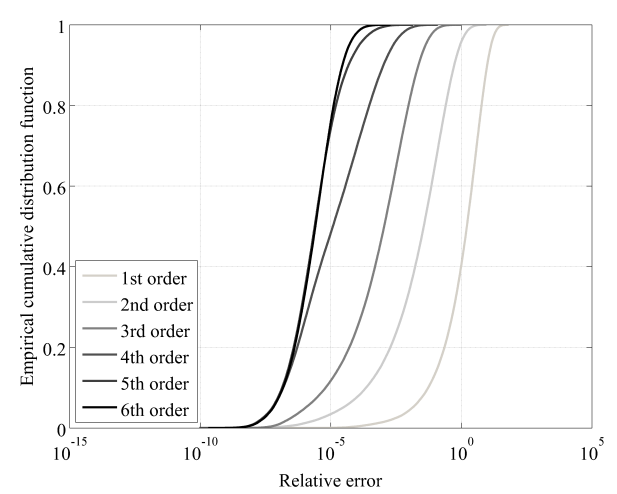

(b) Empirical cumulative distribution function of the relative residuals

Fig. 7: Analysis of the accuracy of the DA method at different expansion orders for optical observations. 


\section{B. Test case B}

The results obtained for the radar observations are analysed with the same approach as for test case A, using again 100,000 normally distributed samples in the observations space. Figure 8 shows the samples as well as their probability, scaled in the range $[0,1]$. In this case the large uncertainties of the instrument pointing angles yield a large uncertainty set in the MEE space. Nevertheless, the normality distribution of the samples is preserved due to the short temporal separation between the observations. 


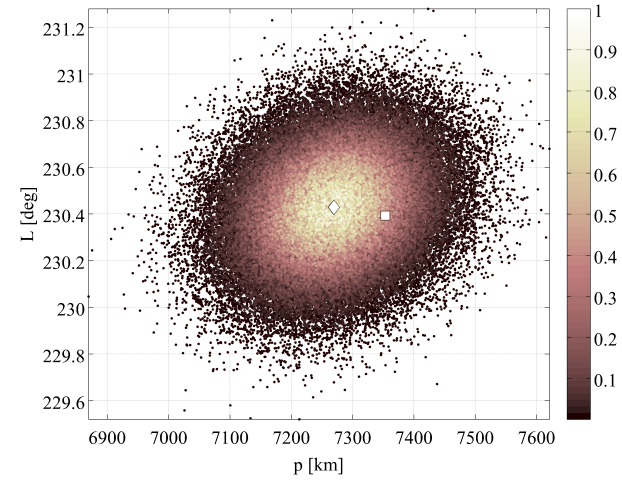

(a)

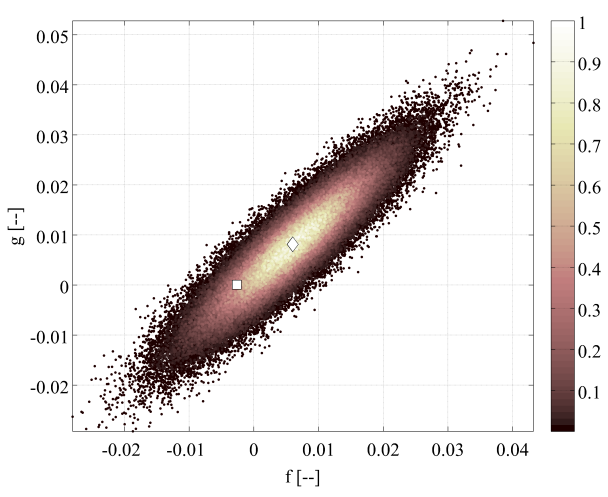

(c)

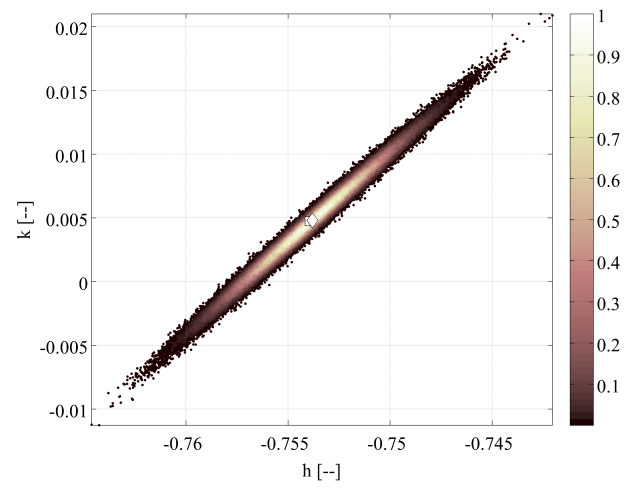

(e)

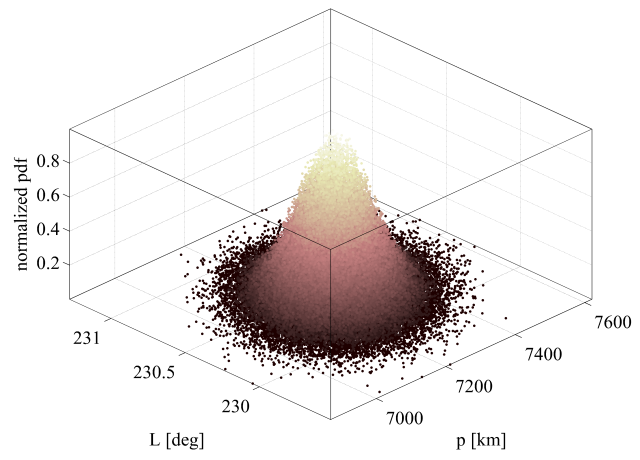

(b)

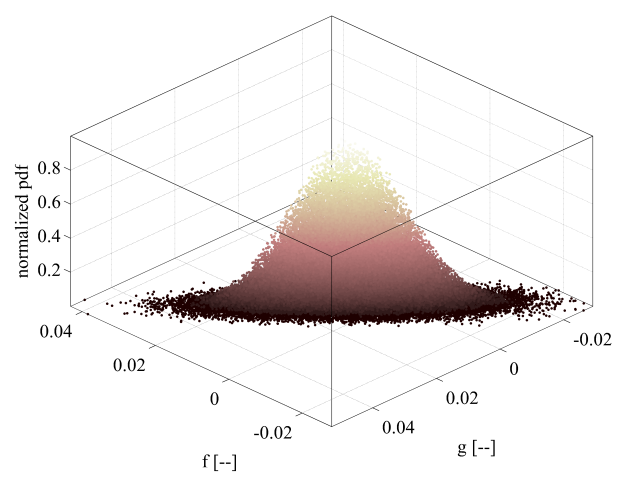

(d)

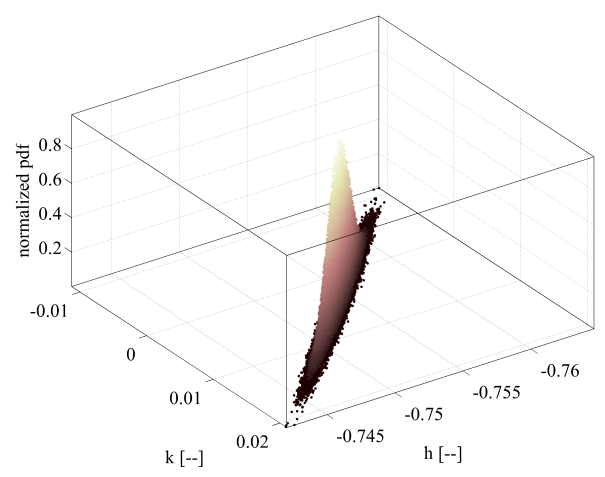

(f)

Fig. 8: Mapped pdf in the MEE space for radar observations

This is clearly confirmed by the analysis of the covariance matrices obtained with 6 -th and 1-st order computations, as reported in Table 5 and 6 . However, similarly to the angles-only IOD, the 6th order expansion is significantly more accurate in representing the O2S map. This is shown in Fig. 9, in which the same analyses of the angles-only case are reported. The estimated truncation errors 
of the state is much smaller for radar observations, as the associated IOD problem is simpler due to the full observation of two position vectors. This is confirmed by Fig. 9(b), in which the empirical cumulative distribution function of the relative residuals is plotted. For the 6-th order expansion, the relative residual is of the order of $1 \times 10^{-7}$, corresponding to a maximum residual of $2.7441 \times 10^{-3}$ arcsec on the angles and $2.1326 \times 10^{-6} \mathrm{~km}$ on the range. As these values are much smaller than the instrument resolution we can conclude that $\mathcal{T}_{\boldsymbol{y}_{1}}(\boldsymbol{\alpha}, \delta, \rho)$ is an accurate approximation of the $\mathrm{O} 2 \mathrm{~S}$ function in the domain of interest.

\begin{tabular}{cccccc}
\hline \hline $\mathrm{p}[\mathrm{km}]$ & $\mathrm{f}$ & $\mathrm{g}$ & $\mathrm{h}$ & $\mathrm{k}$ & $\mathrm{L}[\mathrm{rad}]$ \\
\hline $7.7266 \mathrm{e}+03$ & $-6.7672 \mathrm{e}-01$ & $-8.2801 \mathrm{e}-01$ & $-8.4973 \mathrm{e}-02$ & $-1.0769 \mathrm{e}-01$ & $6.4272 \mathrm{e}-02$ \\
$-9.6537 \mathrm{e}-01$ & $6.3598 \mathrm{e}-05$ & $6.9396 \mathrm{e}-05$ & $4.8983 \mathrm{e}-06$ & $6.2119 \mathrm{e}-06$ & $-1.7431 \mathrm{e}-06$ \\
$-9.8752 \mathrm{e}-01$ & $9.1225 \mathrm{e}-01$ & $9.0989 \mathrm{e}-05$ & $1.0903 \mathrm{e}-05$ & $1.3797 \mathrm{e}-05$ & $-9.6279 \mathrm{e}-06$ \\
$-3.7202 \mathrm{e}-01$ & $2.3637 \mathrm{e}-01$ & $4.3988 \mathrm{e}-01$ & $6.7522 \mathrm{e}-06$ & $9.6472 \mathrm{e}-06$ & $-9.2053 \mathrm{e}-06$ \\
$-3.2775 \mathrm{e}-01$ & $2.0838 \mathrm{e}-01$ & $3.8694 \mathrm{e}-01$ & $9.9317 \mathrm{e}-01$ & $1.3973 \mathrm{e}-05$ & $-1.3320 \mathrm{e}-05$ \\
$2.0246 \mathrm{e}-01$ & $-6.0520 \mathrm{e}-02$ & $-2.7947 \mathrm{e}-01$ & $-9.8088 \mathrm{e}-01$ & $-9.8667 \mathrm{e}-01$ & $1.3044 \mathrm{e}-05$ \\
\hline \hline
\end{tabular}

Table 5: Covariance values between states (upper triangle), correlation values (lower triangle), and variances (diagonal terms) computed at 6-th order for radar observations.

\begin{tabular}{cccccc}
\hline \hline $\mathrm{p}[\mathrm{km}]$ & $\mathrm{f}$ & $\mathrm{g}$ & $\mathrm{h}$ & $\mathrm{k}$ & $\mathrm{L}[\mathrm{rad}]$ \\
\hline $7.7271 \mathrm{e}+03$ & $-6.7678 \mathrm{e}-01$ & $-8.2807 \mathrm{e}-01$ & $-8.4976 \mathrm{e}-02$ & $-1.0770 \mathrm{e}-01$ & $6.4277 \mathrm{e}-02$ \\
$-9.6540 \mathrm{e}-01$ & $6.3600 \mathrm{e}-05$ & $6.9404 \mathrm{e}-05$ & $4.8979 \mathrm{e}-06$ & $6.2123 \mathrm{e}-06$ & $-1.7430 \mathrm{e}-06$ \\
$-9.8753 \mathrm{e}-01$ & $9.1233 \mathrm{e}-01$ & $9.0994 \mathrm{e}-05$ & $1.0904 \mathrm{e}-05$ & $1.3799 \mathrm{e}-05$ & $-9.6289 \mathrm{e}-06$ \\
$-3.7203 \mathrm{e}-01$ & $2.3636 \mathrm{e}-01$ & $4.3992 \mathrm{e}-01$ & $6.7516 \mathrm{e}-06$ & $9.6467 \mathrm{e}-06$ & $-9.2047 \mathrm{e}-06$ \\
$-3.2778 \mathrm{e}-01$ & $2.0839 \mathrm{e}-01$ & $3.8698 \mathrm{e}-01$ & $9.9319 \mathrm{e}-01$ & $1.3973 \mathrm{e}-05$ & $-1.3320 \mathrm{e}-05$ \\
$2.0247 \mathrm{e}-01$ & $-6.0516 \mathrm{e}-02$ & $-2.7950 \mathrm{e}-01$ & $-9.8089 \mathrm{e}-01$ & $-9.8667 \mathrm{e}-01$ & $1.3043 \mathrm{e}-05$ \\
\hline \hline
\end{tabular}

Table 6: Covariance values between states (upper triangle), correlation values (lower triangle), and variances (diagonal terms) computed at 1-st order for radar observations. 


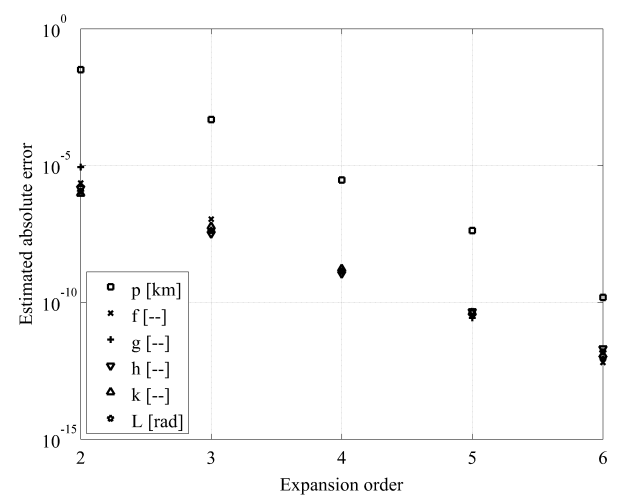

(a) Estimation of the truncation error of $\mathcal{T}_{\boldsymbol{y}_{2}}(\boldsymbol{\alpha}, \boldsymbol{\delta}, \boldsymbol{\rho})$

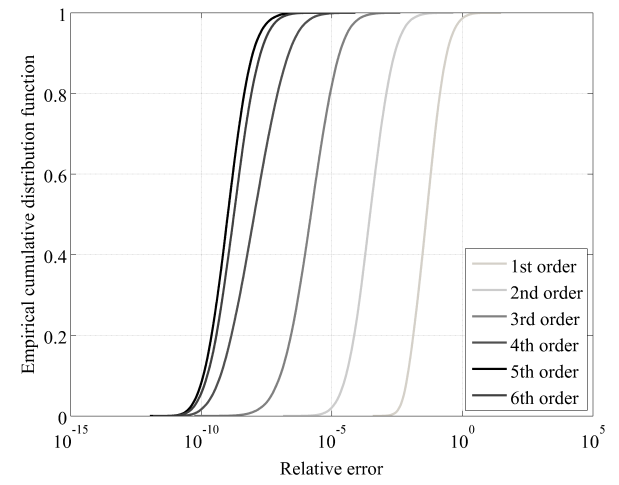

(b) Empirical cumulative distribution function of the relative residuals

Fig. 9: Analysis of the accuracy of the DA method at different expansion orders for radar observations.

As for the optical case, the selection of the set of orbital parameters used for the representation of the spacecraft state plays a key role on the characteristics of the mapped pdf. By comparing Fig. 10(a) with Fig. 10(b), it is clear that, when classical orbital elements are used, the samples are no longer normally distributed in eccentricity, since this orbital parameter cannot take negative values.

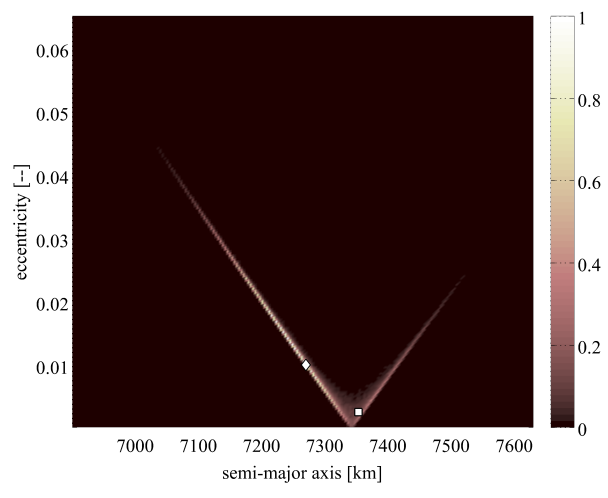

(a) Samples binned in the semi-major axis and eccentricity space at $t_{1}$

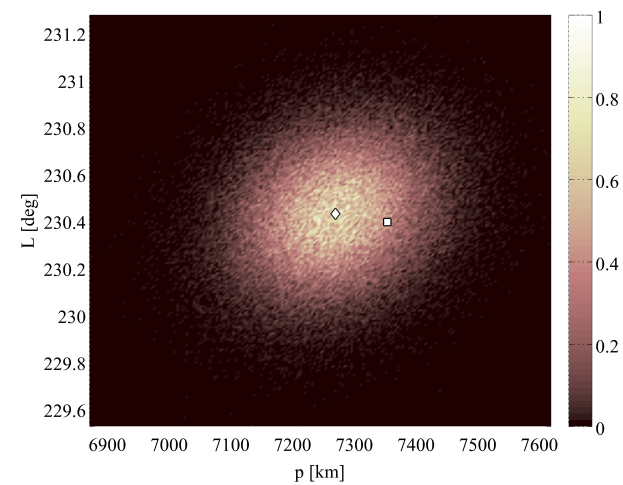

(b) Samples binned in the true longitude and semilatus rectum space at $t_{1}$

Fig. 10: Effect of state representation on the mapped pdf for radar observations.

As already mentioned in Sec. IV B, given the two position vectors and the elapsed time between the observations in the radar observations scenario, a Lambert problem can be solved to compute 
the Taylor approximation of the velocity vectors at the two observation epochs using DA. The solution of the Lambert problem relies on the assumption of a Keplerian motion. Nevertheless, the proposed method can be easily extended to apply to more complex dynamics as described in Sec IV C. This approach is used here to solve the IOD problem by predicting the motion of the object using the numerical propagator AIDA (Accurate Integrator for Debris Analysis [46]), which includes geopotential acceleration, atmospheric drag, solar radiation pressure, and third body gravity as perturbations to the Keplerian motion.

Table 7 compares the mean MEEs at the epoch of the first observation obtained using Kepler's dynamics with the MEEs at the same epoch computed with AIDA. Due to the short time between the two observations, the effect of the perturbations is negligible. This is confirmed by comparing the covariance-correlation matrices of the MEE samples obtained with 6-th order computations in Keplerian dynamics (Table 5) with the one reported in Table 8 and obtained using AIDA.

\begin{tabular}{|c|c|c|c|c|c|c|}
\hline & $\mathrm{p}[\mathrm{km}]$ & $\mathrm{f}$ & $\mathrm{g}$ & $\mathrm{h}$ & $\mathrm{k}$ & $\mathrm{L}$ [rad] \\
\hline Kepler & $7.2699 \mathrm{e}+03$ & $5.9892 \mathrm{e}-03$ & $8.0798 \mathrm{e}-03$ & $-7.5380 \mathrm{e}-01$ & $4.7789 \mathrm{e}-03$ & $1.0305 \mathrm{e}+01$ \\
\hline AIDA & $7.2708 \mathrm{e}+03$ & $5.9534 \mathrm{e}-03$ & $7.9465 \mathrm{e}-03$ & $-7.5381 \mathrm{e}-01$ & $4.76018 \mathrm{e}-03$ & $1.03049 \mathrm{e}+01$ \\
\hline
\end{tabular}

Table 7: Comparison between the mean MEE computed at 6-th order with Keplerian dynamics and AIDA model. 


\begin{tabular}{cccccc}
\hline \hline $\mathrm{p}[\mathrm{km}]$ & $\mathrm{f}$ & $\mathrm{g}$ & $\mathrm{h}$ & $\mathrm{k}$ & $\mathrm{L}[\mathrm{rad}]$ \\
\hline $7.7278 \mathrm{e}+03$ & $-6.7678 \mathrm{e}-01$ & $-8.2818 \mathrm{e}-01$ & $-8.4972 \mathrm{e}-02$ & $-1.0770 \mathrm{e}-01$ & $6.4270 \mathrm{e}-02$ \\
$-9.6536 \mathrm{e}-01$ & $6.3600 \mathrm{e}-05$ & $6.9404 \mathrm{e}-05$ & $4.8965 \mathrm{e}-06$ & $6.2096 \mathrm{e}-06$ & $-1.7406 \mathrm{e}-06$ \\
$-9.8752 \mathrm{e}-01$ & $9.1223 \mathrm{e}-01$ & $9.1011 \mathrm{e}-05$ & $1.0904 \mathrm{e}-05$ & $1.3798 \mathrm{e}-05$ & $-9.6284 \mathrm{e}-06$ \\
$-3.7202 \mathrm{e}-01$ & $2.3630 \mathrm{e}-01$ & $4.3988 \mathrm{e}-01$ & $6.7510 \mathrm{e}-06$ & $9.6459 \mathrm{e}-06$ & $-9.2040 \mathrm{e}-06$ \\
$-3.2775 \mathrm{e}-01$ & $2.0830 \mathrm{e}-01$ & $3.8694 \mathrm{e}-01$ & $9.9317 \mathrm{e}-01$ & $1.3972 \mathrm{e}-05$ & $-1.3319 \mathrm{e}-05$ \\
$2.0244 \mathrm{e}-01$ & $-6.0437 \mathrm{e}-02$ & $-2.7947 \mathrm{e}-01$ & $-9.8088 \mathrm{e}-01$ & $-9.8666 \mathrm{e}-01$ & $1.3042 \mathrm{e}-05$ \\
\hline \hline
\end{tabular}

Table 8: Covariance values between states (upper triangle), correlation values (lower triangle), and variances (diagonal terms) computed at 6-th order when the radar IOD problem is solved with AIDA dynamical model.

The same approach adopted to derive Eq. (43) in test case A can be used here to map the pdf of the measurements at epoch $t_{1}$ into the pdf of the state at epoch $t_{1}+T$, in which $T$ is the period of the nominal solution. Unlike the previous case, to investigate the long-term effects of the perturbations and show the versatility of the proposed method, the pdf is mapped by computing the 6-th order expansion of the flow using AIDA. The result is shown in Figure 11(a) by binning the resulting samples. Once again, the dynamics stretch the set along the orbit and, after one revolution, the set is spread on an arc length of about $30 \mathrm{deg}$. As a clear effect of the increasing nonlinearities, the set starts bending, so drifting apart from the normal distribution. This result is quantified by the covariance of the propagated samples reported in Table 9 , in which the variance of $L$ grows by three orders of magnitude. It is worth to observe that, in contrast with the two-body model, all the elements of the covariance matrices are time varying due to the effect of the perturbations. Lastly, Fig. 11(b) reports the predicted truncation error of $\mathcal{T}_{\boldsymbol{y}_{f}}(\boldsymbol{\alpha}, \boldsymbol{\delta}, \boldsymbol{\rho})$. Due to the large initial domain the accuracy of the Taylor representation of the true longitude quickly degrades, and after one revolution the truncation error at 6-th order increases by six orders of magnitude, passing from $1.52 \times 10^{-12}$ to $2.04 \times 10^{-6} \mathrm{rad}$. What is more important to note is that increasing the expansion order has a small effect on the truncation error, a situation that can be alleviated only by suitably splitting the initial domain [38]. 


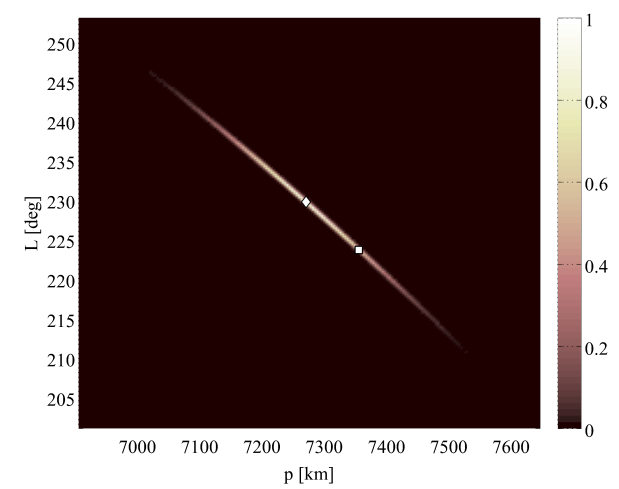

(a) Samples binned in the true longitude and semilatus rectum space after one orbital revolution

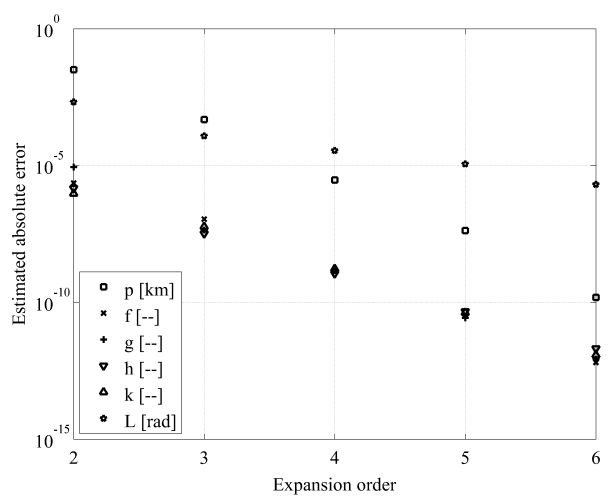

(b) Estimation of the truncation error of $\mathcal{T}_{\boldsymbol{y}_{f}}(\boldsymbol{\alpha}, \boldsymbol{\delta}, \boldsymbol{\rho})$

Fig. 11: Mapped pdf and accuracy analysis of the approximation of the O2S function for radar observations when the solution is propagated forward for one orbit revolution using AIDA dynamical model.

\begin{tabular}{cccccc}
\hline \hline $\mathrm{p}[\mathrm{km}]$ & $\mathrm{f}$ & $\mathrm{g}$ & $\mathrm{h}$ & $\mathrm{k}$ & $\mathrm{L}[\mathrm{rad}]$ \\
\hline $7.7289 \mathrm{e}+03$ & $-6.7687 \mathrm{e}-01$ & $-8.2827 \mathrm{e}-01$ & $-8.4975 \mathrm{e}-02$ & $-1.0769 \mathrm{e}-01$ & $-9.5712 \mathrm{e}+00$ \\
$-9.6516 \mathrm{e}-01$ & $6.3636 \mathrm{e}-05$ & $6.9392 \mathrm{e}-05$ & $4.9001 \mathrm{e}-06$ & $6.2141 \mathrm{e}-06$ & $8.4213 \mathrm{e}-04$ \\
$-9.8744 \mathrm{e}-01$ & $9.1171 \mathrm{e}-01$ & $9.1033 \mathrm{e}-05$ & $1.0902 \mathrm{e}-05$ & $1.3795 \mathrm{e}-05$ & $1.0230 \mathrm{e}-03$ \\
$-3.7197 \mathrm{e}-01$ & $2.3639 \mathrm{e}-01$ & $4.3973 \mathrm{e}-01$ & $6.7523 \mathrm{e}-06$ & $9.6473 \mathrm{e}-06$ & $9.6687 \mathrm{e}-05$ \\
$-3.2770 \mathrm{e}-01$ & $2.0839 \mathrm{e}-01$ & $3.8680 \mathrm{e}-01$ & $9.9317 \mathrm{e}-01$ & $1.3974 \mathrm{e}-05$ & $1.2089 \mathrm{e}-04$ \\
$-9.9900 \mathrm{e}-01$ & $9.6869 \mathrm{e}-01$ & $9.8382 \mathrm{e}-01$ & $3.4143 \mathrm{e}-01$ & $2.9676 \mathrm{e}-01$ & $1.1876 \mathrm{e}-02$ \\
\hline \hline
\end{tabular}

Table 9: Covariance values between states (upper triangle), correlation values (lower triangle), and variances (diagonal terms) computed at 6-th order for the radar IOD problem when the solution is propagated with the AIDA dynamical model for one revolution.

\section{Computational Times}

The simulations presented in this work were run on a MacBook Air with a 1.8 GHz Intel Core i5 processor and 4 GB $1600 \mathrm{MHz}$ memory. In Table 10 a comparison between the CPU times required for a DA-based MC and a pointwise MC is provided for both Keplerian and AIDA dynamical models. 
As expected both the optical and radar 6-th order solutions require more CPU time with respect to a classical pointwise solution. In addition, the optical IOD problem is more time consuming than the radar one, as the latter requires only the solution of one Lambert problem. However, the time required for the evaluation of a 6 -th order map is always (independently from the dynamical system used) lower than a pointwise solution. As a result, a DA-based MC analysis is in all cases more efficient when the number of samples to be evaluated is greater than 312 . The situation becomes much more in favour of the DA approach when a more complex dynamical system is used as shown by the AIDA column in the table. It is worth noting that the availability of the DA expansion of the O2S function enables the computation of both the mapped pdf and its statistical moments without samples.

\begin{tabular}{lccc}
\hline \hline & \multicolumn{2}{c}{ Keplerian } & AIDA \\
& Optical & Radar & Radar \\
\hline DA-based IOD solution & 1.186 & $6.295 \times 10^{-2}$ & 31.994 \\
Single DA map evaluation & $1.943 \times 10^{-4}$ & $9.046 \times 10^{-5}$ & $3.225 \times 10^{-4}$ \\
Pointwise IOD solution & $4.001 \times 10^{-3}$ & $3.110 \times 10^{-4}$ & 0.564 \\
MC break even point & 312 & 286 & 57 \\
\hline \hline
\end{tabular}

Table 10: Comparison between the CPU times (in seconds) between 6-th order DA computations and pointwise computations.

\section{Conclusions}

A general method for mapping a pdf from the observations space into the object orbital space in the frame of the solution of an IOD problem has been presented. This method is based on the high order Taylor expansion of both the O2S map and its Jacobian obtained using DA techniques. A simple Cartesian to polar transformation has been used as illustrative example to show how the method works in a problem in which the analytical solution is available. Then, the approach was run on two IOD scenarios: an optical observation of a spacecraft in GEO and a radar observation of a spent rocket in LEO. It was shown that DA can be effectively used to nonlinearly map pdfs (and compute their statistical moments), thus enabling a probabilistic analysis of IOD problems 
without the need of samples. The availability of the Taylor expansion of the O2S map enables also the execution of DA-based MC simulations. These are in general more efficient than classical MC, but one needs to accept a loss in accuracy. However, it was shown that observation residuals associated with 6-th order computations were orders of magnitude smaller than the resolution of both optical and radar instruments. Finally, the method is independent of the model used to describe the spacecraft dynamics, as well as of the pdf adopted for the measurements. For these reasons the proposed approach has the potential to be used in data association problems, where the capability of attributing a statistical meaning to the IOD solutions is a key.

\section{Acknowledgments}

R. Armellin acknowledges the support received by the Marie Sklodowska-Curie grant 627111 (HOPT - Merging Lie perturbation theory and Taylor Differential algebra to address space debris challenges). This work has been partially funded by the Spanish Finance and Competitiveness Ministry under Project ESP2014-57071-R.

\section{References}

[1] Wilden, H., Kirchner, C., Peters, O., Bekhti, N. B., Brenner, A., and Eversberg, T., "GESTRAÑA phased-array based surveillance and tracking radar for space situational awareness," Phased Array Systems and Technology (PAST), 2016 IEEE International Symposium on, IEEE, 2016, pp. 1-5.

[2] Pechkis, D. L., Pacheco, N. S., and Botting, T. W., "Statistical approach to the operational testing of space fence," IEEE Aerospace and Electronic Systems Magazine, Vol. 31, No. 11, 2016, pp. 30-39.

[3] Schumacher, P. W., Sabol, C., Higginson, C. C., and Alfriend, K. T., "Uncertain Lambert Problem," Journal of Guidance, Control, and Dynamics, 2015, pp. 1-12.

[4] Gauss, C. F., Theoria Motus Corporum Coelestium in Sectionibus Conicis Solem Ambientium, sumtibus Frid. Perthes et IH Besser, 1809.

[5] Laplace, P., "Mémoires de l'Académie Royale des Sciences," Paris, Reprinted in Laplace's Collected Works, Vol. 10, 1780.

[6] Merton, G., "A modification of Gauss's method for the determination of orbits," Monthly Notices of the Royal Astronomical Society, Vol. 85, 1925, pp. 693. 
[7] Celletti, A. and Pinzari, G., "Dependence on the observational time intervals and domain of convergence of orbital determination methods," Periodic, Quasi-Periodic and Chaotic Motions in Celestial Mechanics: Theory and Applications, Springer, 2006, pp. 327-344.

[8] Gronchi, G. F., "Multiple solutions in preliminary orbit determination from three observations," Celestial Mechanics and Dynamical Astronomy, Vol. 103, No. 4, 2009, pp. 301-326.

[9] Escobal, P. R., "Methods of orbit determination," New York: Wiley, 1965, Vol. 1, 1965.

[10] Gooding, R., "A new procedure for the solution of the classical problem of minimal orbit determination from three lines of sight," Celestial Mechanics and Dynamical Astronomy, Vol. 66, No. 4, 1996, pp. 387423.

[11] Hussein, I. I., Roscoe, C. W., Wilkins, M. P., and Schumacher, P. W., "On mutual information for observation-to-observation association," Information Fusion (Fusion), 2015 18th International Conference on, IEEE, 2015, pp. 1293-1298.

[12] Milani, A., Gronchi, G. F., Vitturi, M. d., and Knežević, Z., "Orbit determination with very short arcs. I admissible regions," Celestial Mechanics and Dynamical Astronomy, Vol. 90, No. 1, 2004, pp. 57-85.

[13] Roscoe, C., Hussein, I., Wilkins, M., and Schumacher, P., "The Probabilistic Admissible Region with Additional Constraints," Proceedings of the Advanced Maui Optical and Space Surveillance Technologies Conference, held in Wailea, Maui, Hawaii, September 15-18, 2014, Ed.: S. Ryan, The Maui Economic Development Board, id. 91, Vol. 1, 2015, p. 91.

[14] Worthy III, J. L. and Holzinger, M. J., "Incorporating uncertainty in admissible regions for uncorrelated detections," Journal of Guidance, Control, and Dynamics, Vol. 38, No. 9, 2015, pp. 1673-1689.

[15] Battin, R., An Introduction to the Mathematics and Methods of Astrodynamics, AIAA Education Series, Reston, VA, 1999.

[16] Gooding, R., "A procedure for the solution of Lambert's orbital boundary-value problem," Celestial Mechanics and Dynamical Astronomy, Vol. 48, No. 2, 1990, pp. 145-165.

[17] Arora, N. and Russell, R., "A fast and robust multiple revolution Lambert algorithm using a cosine transformation," Paper AAS, Vol. 13-728, 2013.

[18] Muinonen, K. and Bowell, E., "Asteroid orbit determination using Bayesian probabilities," Icarus, Vol. 104, No. 2, 1993, pp. 255-279.

[19] Virtanen, J., Muinonen, K., and Bowell, E., "Statistical ranging of asteroid orbits," Icarus, Vol. 154, No. 2, 2001, pp. 412-431.

[20] Binz, C. R. and Healy, L. M., "Uncertainty Characterization for Angles-Only Initial Orbit Determination," Advances in the Astronautical Sciences, Vol. 150, 2014, pp. 1777-1792. 
[21] Roscoe, C. W., Hussein, I. I., Schumacher, P. W., and Wilkins, M. P., "On Uncertain Angles-Only Track Initiation for SSA," AIAA/AAS Astrodynamics Specialist Conference, 2014, p. 4468.

[22] Hussein, I. I., Roscoe, C. W., Wilkins, M. P., and Schumacher Jr, P. W., "Probabilistic admissibility in angles-only initial orbit determination," Proceedings of the 24 th International Symposium on Space Flight Dynamics, 2014, pp. 5-9.

[23] Weisman, R., Majji, M., and Alfriend, K., "Analytic characterization of measurement uncertainty and initial orbit determination on orbital element representations," Celestial Mechanics and Dynamical Astronomy, Vol. 118, No. 2, 2014, pp. 165-195.

[24] Weisman, R. and Jah, M., "Uncertainty quantification for angles-only initial orbit determination," AAS/AIAA Spaceflight Mechanics Meeting, Santa Fe, AAS, 2014, pp. 14-434.

[25] Berz, M., Differential Algebraic Techniques, Entry in Handbook of Accelerator Physics and Engineering, World Scientific, New York, 1999a.

[26] Park, R. and Scheeres, D., "Nonlinear Mapping of Gaussian Statistics: theory and Applications to Spacecraft trajectory Design," Journal of Guidance, Control and Dynamics, Vol. 29, No. 6, 2006.

[27] Valli, M., Armellin, R., Di Lizia, P., and Lavagna, M., "Nonlinear mapping of uncertainties in celestial mechanics," Journal of Guidance, Control, and Dynamics, Vol. 36, No. 1, 2012, pp. 48-63.

[28] Soong, T., Fundamentals of Probability and Statistics for Engineers, Vol. 50, John Wiley \& Sons, 2004.

[29] Wittig, A., Colombo, C., and Armellin, R., "Long-term density evolution through semi-analytical and differential algebra techniques," Celestial Mechanics and Dynamical Astronomy, 2017, pp. 1-18.

[30] Berz, M., The new method of TPSA algebra for the description of beam dynamics to high orders, Los Alamos National Laboratory, 1986, Technical Report AT-6:ATN-86-16.

[31] Berz, M., "The method of power series tracking for the mathematical description of beam dynamics," Nuclear Instruments and Methods A258, 1987.

[32] Berz, M., Modern Map Methods in Particle Beam Physics, Academic Press, 1999b.

[33] Berz, M. and Makino, K., COSY INFINITY version 9 reference manual, Michigan State University, East Lansing, MI 48824, 2006, MSU Report MSUHEP060803.

[34] Rasotto, M., Morselli, A., Wittig, A., Massari, M., Di Lizia, P., Armellin, R., Valles, C., and Ortega, G., "Differential Algebra Space Toolbox for Nonlinear Uncertainty Propagation in Space Dynamics," 6th International Conference on Astrodynamics Tools and Techniques, ICATT 2016. Proceedings of the, 2016, pp. 1-11.

[35] Di Lizia, P., Armellin, R., and Lavagna, M., "Application of high order expansions of two-point boundary value problems to astrodynamics," Celestial Mechanics and Dynamical Astronomy, Vol. 102, No. 4, 
2008, pp. $355-375$.

[36] Kaplan, M. H., Modern spacecraft dynamics and control, Vol. 1, John Wiley and Sons, New York, NY, 1976.

[37] Prussing, J. E. and Conway, B. A., Orbital mechanics, Oxford University Press, 1993.

[38] Wittig, A., Di Lizia, P., Armellin, R., Makino, K., Bernelli-Zazzera, F., and Berz, M., "Propagation of large uncertainty sets in orbital dynamics by automatic domain splitting," Celestial Mechanics and Dynamical Astronomy, Vol. 122, No. 3, 2015, pp. 239-261.

[39] Makino, K., Rigorous analysis of nonlinear motion in particle accelerators, Ph.D. thesis, Michigan State University, 1998.

[40] Isserlis, L., "On a formula for the product-moment coefficient of any order of a normal frequency distribution in any number of variables," Biometrika, Vol. 12, No. 1 and 2, 1918.

[41] Kan, R., "From moments of sum to moments of product," Journal of Multivariate Analysis, Vol. 99, No. 3, 2008.

[42] Armellin, R., Lizia, P. D., Bernelli-Zazzera, F., Berz, M., and Di Lizia, P., "Asteroid close encounters characterization using differential algebra: the case of Apophis," Celestial Mechanics and Dynamical Astronomy, Vol. 107, No. 4, 2010, pp. 451-470.

[43] Armellin, R., Di Lizia, P., and Lavagna, M., "High-order expansion of the solution of preliminary orbit determination problem," Celestial Mechanics and Dynamical Astronomy, Vol. 112, No. 3, 2012, pp. 331-352.

[44] Armellin, R., Di Lizia, P., and Zanetti, R., "Dealing with uncertainties in angles-only initial orbit determination," Celestial Mechanics and Dynamical Astronomy, Vol. 125, No. 4, 2016, pp. 435-450.

[45] Armellin, R., Di Lizia, P., Topputo, F., Lavagna, M., Bernelli-Zazzera, F., and Berz, M., "Gravity assist space pruning based on differential algebra," Celestial mechanics and dynamical astronomy, Vol. 106, No. 1, 2010, pp. 1-24.

[46] Morselli, A., Armellin, R., Di Lizia, P., and Bernelli-Zazzera, F., "A High Order Method for Orbital Conjunctions Analysis: Sensitivity to Initial Uncertainties," Advances in Space Research, Vol. 53, No. 3, 2014, pp. 490-508. 